\title{
Verteilungspolitische Interventionen
}

\section{Eingriff in die Primärverteilung vs. Korrektur der Marktergebnisse}

\section{Hagen Krämer}

Online publiziert: 21. Oktober 2020

(C) Der/die Autor(en) 2020

Zusammenfassung Der Beitrag beschäftigt sich mit der Frage, auf welche Art und Weise die Verteilungspolitik in der heutigen Zeit ausgestaltet werden kann. Welches sind denkbare Ziele, welches mögliche Instrumente von verteilungspolitischen Interventionen in einer marktwirtschaftlichen Ordnung? Sind weitgehende Eingriffe in Marktprozesse unvermeidbar, wenn die Ungleichheit der Einkommensverteilung reduziert werden soll? Oder sollten diese weitgehend vermieden werden?

Zur Beantwortung dieser Fragen wird zunächst ein kurzer Überblick über wesentliche Denkrichtungen in den Wirtschaftswissenschaften gegeben, die sich damit beschäftigt haben, ob und wie stark der Staat die Einkommensverteilung beeinflussen sollte. Danach werden verschiedene Kriterien für distributive Gerechtigkeit besprochen. Anschließend wird ein vereinfachtes Schema für verschiedene Verteilungstypologien entwickelt, das unterschiedliche Vorstellungen von distributiver Gerechtigkeit abbilden kann. Abschließend wird der Versuch einer neuen Systematisierung der Distributionspolitik vorgestellt, mit der eine Erweiterung des üblichen Schemas „Primär- versus Sekundärverteilung“ vorgenommen wird.

Schlüsselwörter Primär- und Sekundärverteilung · Verteilungspolitik · Einkommensumverteilung · Philosophie der Einkommensverteilung · Allokatives und normatives Marktversagen

Schriftliche Fassung eines Referats beim Wirtschaftspolitischen Ausschuss des Vereins für Socialpolitik, Berlin, 4.-5. März 2020

H. Krämer $(\bowtie)$

Fakultät für Wirtschaftswissenschaften, Hochschule Karlsruhe - Technik und Wirtschaft, Moltkestraße 30, 76133 Karlsruhe, Deutschland

E-Mail: hagen.kraemer@hs-karlsruhe.de 


\section{Equality-enhancing policy interventions}

Intervening in primary income distribution vs. correction of market results

Abstract This article deals with the question of how distribution policy can be shaped in the contemporary world. What are conceivable goals, what possible instruments of distributional policy interventions in today's market economies? Are extensive interventions in market processes unavoidable if the inequality of income distribution is to be reduced? Or should they be largely avoided?

In order to answer these questions, a brief overview is first given of major schools of thought in economics that have dealt with whether and to what extent the state should influence income distribution. Then, different criteria for distributive justice are discussed. Subsequently, a simplified scheme for different distributional typologies is developed, which can represent different notions of distributive justice. Finally, the attempt to systematize distribution policy in a new way is presented, which is an extension of the usual scheme of "primary versus secondary income distribution".

Keywords Primary and secondary income distribution · Distribution policy · Income redistribution - Philosophy of income distribution · Allocative and normative market failure

\section{Einleitung}

Das Ziel, eine ,gerechte“ oder „faire“ Verteilung von Einkommen und Vermögen anzustreben, gehört nicht zu den vier wirtschaftspolitischen Zielen, die im „Gesetz zur Förderung der Stabilität und des Wachstums der Wirtschaft (StabG)“ vom 8. Juni 1967 enthalten sind. Dennoch ist die Verteilungspolitik in Deutschland wie in anderen Ländern auch ein essenzielles Teilgebiet der praktischen Wirtschaftspolitik. Für Deutschland kann dies nicht zuletzt daraus abgeleitet werden, dass im „Gesetz über die Bildung eines Sachverständigenrats zur Begutachtung der gesamtwirtschaftlichen Entwicklung“ vom 14. August 1963 festgelegt wurde, dass der Sachverständigenrat auch die Bildung und Verteilung von Einkommen und Vermögen ,in seine Untersuchungen einbeziehen“ soll (ebd. § 2, Abs. 2 (1)). Gleichzeitig gehört die Verteilungspolitik mit zu den besonders kontrovers diskutierten Themen der Wirtschaftspolitik. Zum einen berührt sie unmittelbar die seit jeher stark umstrittene Frage nach dem Ausmaß und der Intensität von Staatseingriffen in die Wirtschaft. Hier liegen die Ansichten zwischen den verschiedenen „Schulen“ der wirtschaftswissenschaftlichen Theorie teilweise weit auseinander. Zum anderen lassen sich Werturteile bei der Diskussion um die ,richtige“ oder ,gerechte“ Verteilung nicht vermeiden. Da sich die moderne Wirtschaftswissenschaft jedoch als eine positive Wissenschaft sieht, werden normative Verteilungsfragen in der Ökonomik kaum behandelt.

Durch die in praktisch allen Industrieländern in den letzten Dekaden gestiegene Ungleichheit in der Verteilung der Einkommen und Vermögen hat die öffentliche und wissenschaftliche Debatte über die Verteilungs- und Umverteilungspolitik wieder an 
Fahrt aufgenommen. Umstritten ist dabei zum einem, ob die Ungleichheit so stark zugenommen hat, dass es zusätzlicher verteilungspolitischer Maßnahmen des Staates bedarf. Zum anderen herrscht Uneinigkeit darüber, was die richtigen Ansatzpunkte und Instrumente der Verteilungspolitik sein sollten.

Im vorliegenden Beitrag steht die Frage, ob die Ungleichheit in der Einkommensverteilung in Deutschland oder anderen Ländern so stark zugenommen hat, dass ein verteilungspolitischer Handlungsbedarf besteht, nicht im Vordergrund. Dieses Thema wurde unter anderem auch in dieser Zeitschrift vor nicht allzu langer Zeit ausführlich behandelt (Klodt 2018). Ich möchte mich hier mit der Frage nach möglichen, sinnvollen und legitimen Ansatzpunkten und Instrumenten einer Politik der Verteilung und Umverteilung befassen. Es wird im Folgenden daher angenommen, dass die politisch Verantwortlichen die Absicht verfolgen, die Einkommensverteilung gegenüber dem Status quo gleichmäßiger werden zu lassen. Damit stellt sich die Frage, welche Instrumente hierfür besonders gut geeignet erscheinen. Unter anderem gilt es herauszufinden, welche verteilungspolitischen Maßnahmen zielgenau und effektiv sind und welches gegebenenfalls problematische Effekte sind, die aus ihrem Einsatz resultieren können.

Beschäftigt man sich näher mit diesen Fragen, dann fällt auf, dass in letzter Zeit ein gewisser Perspektivwechsel in der Umverteilungsdebatte stattgefunden hat. Bis vor kurzem schien ein weitgehender Konsens unter den Ökonominnen und Ökonomen zu bestehen, dass staatliche Eingriffe in Marktprozesse mit dem Ziel, eine Veränderung der Einkommensverteilung herbeizuführen, möglichst vermieden werden sollten. Derartige Markteingriffe - so die weitverbreitete Überzeugung - würden die Fähigkeit von Wettbewerbsmärkten, die größtmögliche Effizienz bei der Ressourcenallokation zu erbringen, einschränken oder sogar zerstören. Daher lautete die verbreitete Empfehlung, die Verteilung von Einkommen und Vermögen - wenn überhaupt - vorwiegend durch redistributive Maßnahmen zu modifizieren, anstatt zu versuchen, die Verteilung durch Eingriffe in den Marktprozess zu beeinflussen. Diese Sichtweise wird jedoch zunehmend infrage gestellt. Der in Deutschland im Jahr 2015 eingeführte allgemeine gesetzliche Mindestlohn ist ein markantes Beispiel eines Eingriffs von Seiten der Politik in die Arbeitsmärkte für Niedriglohnempfänger. Aber nicht nur in der Politik, sondern auch in der Wissenschaft mehren sich die Stimmen, die nicht in erster Linie mittels der Sekundärverteilung eine gleichmäßigere Einkommensverteilung erreichen wollen. Dabei sind allerdings die Begründungen hierfür recht verschieden. Marcel Fratzscher (2016) vertritt die Auffassung, dass die Umverteilung in Deutschland bereits ein so großes Ausmaß angenommen habe, dass weitere Umverteilungsmaßnahmen weder sinnvoll noch durchsetzbar seien. Fratzscher sieht stattdessen praktisch ausschließlich die Bildungspolitik in der Pflicht, Einkommensungleichheiten durch höhere Chancengleichheit zu reduzieren. Nach Ansicht von Jens Südekum (2018) sollte die Wirtschaftspolitik angesichts der durch die Digitalisierung zukünftig zu erwartenden negativen Verteilungseffekte auf mehreren Feldern vorwiegend die Primärverteilung der Markteinkommen in den Fokus nehmen. Auch in der internationalen Fachwelt lassen sich zunehmend Auffassungen finden, die in dieselbe Richtung gehen. Branko Milanović (2016) sieht in einer globalisierten Welt kaum noch Möglichkeiten, höhere Steuern für gutverdienende international mobile Arbeitskräfte sowie generell auf Kapitalerträge zu erheben und 
setzt daher ganz auf Maßnahmen, die die Primärverteilung beeinflussen. Der international renommierte Verteilungsforscher Tony Atkinson (2015) sah dagegen die Möglichkeiten einer Umverteilung von Einkommen durch eine progressive Einkommensteuer nicht für erschöpft an und sprach sich sogar dezidiert für eine größere Steuerprogression aus. Aber auch Atkinson hielt ergänzend zur klassischen Umverteilungspolitik eine Reihe von Maßnahmen, die unmittelbar die Primärverteilung beeinflussen, für unabdingbar, wenn man die Einkommensungleichheit erfolgreich verringern wolle. Auch in dem weltweit stark beachteten Buch zur ökonomischen Ungleichheit von Thomas Piketty (2014) wird an mehreren Stellen hervorgehoben, dass durch die Politik die Balance der Kräfteverhältnisse zwischen Arbeit und Kapital wiederhergestellt werden müsse, um die Einkommen wieder gleichmäßiger zu verteilen. In die gleiche Richtung argumentiert Richard Freeman (2020), der sich im Hinblick auf die Situation in den USA dafür ausspricht, durch gesetzgeberische Maßnahmen die Verhandlungsmacht der Gewerkschaften zu stärken und die Arbeitnehmer stärker an den Unternehmen zu beteiligen. Jüngst setzten sich Olivier Blanchard und Dani Rodrik für einen multi-instrumentellen Ansatz zur Verringerung der Einkommensungleichheit ein, der ebenfalls eine Reihe von Eingriffen in Marktprozesse einschließt (Blanchard und Rodrik 2019, 2021; Rodrik 2019a, b). Auch wenn all den genannten Ökonomen mögliche negative Anreizeffekte und Effizienzkosten von staatlichen Eingriffen in die Einkommensverteilung bewusst sind, halten sie offenbar die Probleme, die aus der zunehmenden Ungleichheit entstehen oder ihrer Ansicht nach noch entstehen werden, für so gravierend, dass sie weitgehende verteilungspolitische Interventionen fordern, die über die üblichen Instrumente zur Korrektur der Marktergebnisse hinausgehen. Sie sprechen sich daher teilweise explizit und teilweise implizit für Eingriffe in die Bildung der Primäreinkommen und/ oder für staatliche Maßnahmen zur Veränderung der Ausstattung der Marktakteure und der Gestaltung von Institutionen aus.

Der vorliegende Beitrag will sich mit den Hintergründen dieser neuen Vorschläge auseinandersetzen. Dabei steht hier die personelle Einkommensverteilung im Vordergrund. Die Ungleichheit der Vermögensverteilung sowie Fragen nach der inter- und intragenerativen Verteilung durch Staatsverschuldung, Geldpolitik etc. können hier nicht behandelt werden. Ebenso wenig kann auf die Verteilungswirkungen von öffentlichen Ausgaben eingegangen werden. Auch die unterschiedlichen Instrumente, Akteure und Ziele von Umverteilungspolitik können allenfalls am Rande angesprochen werden.

Der nachfolgende Text ist wie folgt aufgebaut: Zunächst sollen einige empirische Trends der Einkommensverteilung in Deutschland und einigen anderen ausgewählten Ländern dargestellt werden. Dabei geht es vor allem um die Frage, ob sich in der jüngeren Vergangenheit das Ausmaß der monetären Einkommensumverteilung geändert hat. Diesbezügliche Trends werden im Wesentlichen durch einen Vergleich zwischen der Veränderung in der Verteilung der Primäreinkommen und der Sekundäreinkommen dargestellt. Im darauffolgenden Abschnitt soll der Frage nachgegangen werden, welche Auffassung verschiedene Denkrichtungen in den Wirtschaftswissenschaften hinsichtlich der Frage vertreten, ob der Staat die Einkommensverteilung beeinflussen sollte und welche Verteilungsziele dabei gegebenenfalls verfolgt werden sollten. Anschließend setze ich mich mit zwei verschiedenen Konzepten des 
Marktversagens auseinander: dabei wird dem verbreiteten Konzept des allokativen Marktversagens das Konzept des normativen Marktversagens gegenübergestellt. Im vorletzten Abschnitt dieser Arbeit werden verschiedene Verteilungstypologien für die personelle Einkommensverteilung entwickelt, wodurch eine normative Orientierungshilfe für die Beurteilung einer gesellschaftlich erwünschten Verteilung von Einkommen entstehen soll. Auf dieser Basis wird auch ein Vorschlag für eine Einkommensverteilung aufgestellt, wie sie im Rahmen einer sozialen Marktwirtschaft angebracht sein könnte. Im vorletzten Kapitel steht der Versuch einer Systematisierung der Distributionspolitik im Mittelpunkt, mit der eine Erweiterung des üblichen Schemas Primär- versus Sekundärverteilung vorgestellt wird. Dabei wird auch darauf eingegangen, welche geeigneten verteilungspolitischen Empfehlungen dieses Schema für die Praxis bietet. Der Beitrag endet mit einigen abschließenden Bemerkungen zu den Perspektiven der Verteilungspolitik.

\section{Entwicklungen in der Einkommensverteilung}

Bei der Analyse der Verteilung der Einkommen auf Personen bzw. Haushalte wird üblicherweise auf zwei verschiedene Einkommenskonzepte abgestellt: die Primärund die Sekundärverteilung von Einkommen. Bei der Primärverteilung betrachtet man die Einkommen, die unmittelbar aus dem Marktprozess hervorgegangen sind. Hierzu zählen Einkommen aus selbstständiger und unselbstständiger Arbeit sowie Einkommen aus Vermögen und unternehmerischer Tätigkeit. Obwohl der Staat und andere Institutionen durch die Setzung von Rahmenbedingungen und Eingriffe in Märkte die Einkommensbildung beeinflussen, geht man bei der Primärverteilung davon aus, dass diese im Wesentlichen aus dem Marktprozess heraus entsteht. Badelt und Österle (2001, S. 54f.) sprechen von originärer Primärverteilung, wenn die Verteilung sich ohne jeglichen staatlichen Einfluss ergibt. Dies ist allerdings eine hypothetische Ausgangslage, die sich in der Realität so nicht wiederfindet. Denn auch die Primärverteilung ergibt sich durch Handlungen der Wirtschaftssubjekte, die im Rahmen einer staatlichen Ordnung agieren und eine Vielzahl von staatlichen Aktivitäten antizipieren, wie etwa die Besteuerung der Markteinkommen oder arbeitsmarktpolitische Maßnahmen. Badelt und Österle (ebd.) bezeichnen eine solche Verteilung daher als intermediäre Primärverteilung. Die intermediäre Primärverteilung kann durch eine Vielzahl von Instrumenten, wie Regulierungen, öffentlich bereitgestellte Güter, Lohn- und Preispolitik sowie Bildungs- und Vermögenspolitik, beeinflusst werden. In diesem Sinne kann jede staatliche Aktivität - unabhängig davon, ob sie verteilungspolitische Intentionen hat oder nicht - Verteilungswirkungen entfalten (ebd., S. 50). Bei der Sekundärverteilung hingegen werden die zahlreichen Maßnahmen des Staates zur Einkommensumverteilung explizit mitberücksichtigt. Dazu gehören einerseits Einkommenszuflüsse, die ein Haushalt erhält; bedeutsam sind hier Transfereinkommen wie zum Beispiel Renten, Arbeitslosenunterstützung, Kindergeld und vieles mehr. Andererseits verringern diverse Einkommensabflüsse das verfügbare Einkommen eines Haushaltes; hierzu gehören vor allem Sozialbeiträge und direkte Steuern. Wenn man von den Markteinkommen den Saldo aus Steuern und Transfers abzieht, erhält man die verfügbaren Einkommen (auch als 


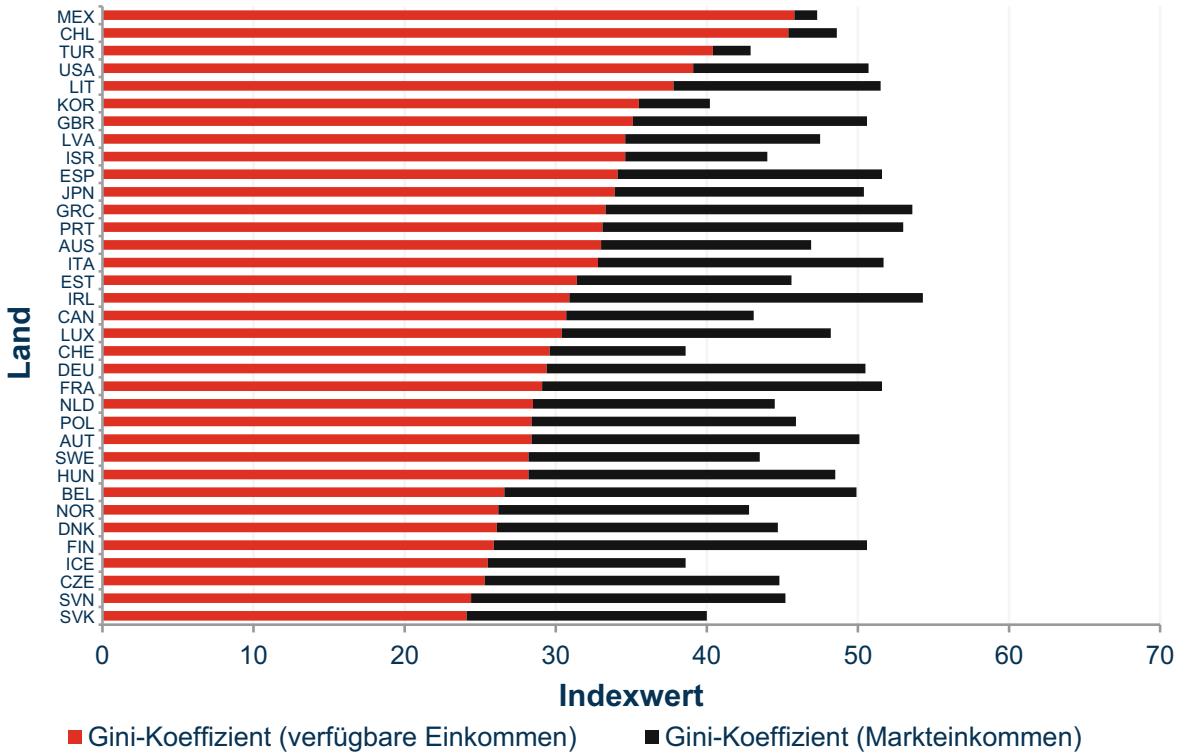

Abb. 1 Gini-Koeffizienten der Einkommensverteilung in OECD-Ländern, 2016 (CHE, CHL, ICE, JPN, TUR: 2015) (Einkommen beziehen sich auf bedarfsgewichtete Haushaltseinkommen. Balkendiagramme überlappen und beginnen jeweils bei Null. Beispiel: Der Gini-Koeffizient der Markteinkommen beträgt in Dänemark ca. 45, der Gini-Koeffizient der verfügbaren Einkommen ca. 26. Gini-Koeffizient: $0=$ völlige Gleichverteilung der Einkommen, 100 = völlige Ungleichverteilung der Einkommen). (Quelle: OECD 2020; Abruf am 04.01.2020)

Nettoeinkommen bezeichnet). Relevanter als die Markteinkommen ist für die privaten Haushalte aus ökonomischer Sicht letztendlich das verfügbare Einkommen, da dieses die Konsum- und Sparmöglichkeiten bestimmt.

\subsection{Einkommensverteilung und -umverteilung nach dem Gini-Koeffizienten}

Bei einem internationalen Vergleich zeigt sich, dass Deutschland bei der Ungleichheit der Markteinkommen mit einem Gini-Index von 50,4 Punkten in 2016 mit an der Spitze aller OECD-Länder liegt (vgl. Abb. 1).

Ähnlich hohe Gini-Koeffizienten weisen außerdem noch Italien und Frankreich auf, während die Markteinkommen gemessen am Gini-Koeffizienten in Kanada und in Schweden deutlich gleichmäßiger verteilt sind. In allen OECD-Ländern ist die Ungleichheit der verfügbaren Einkommen geringer als die der Markteinkommen. Dies zeigt sich an den jeweiligen roten Balken, die den Gini-Koeffizienten der verfügbaren Einkommen wiedergeben. Dies bedeutet, dass die staatlichen Maßnahmen der Einkommensumverteilung überall dazu beitragen, die Ungleichheit der Einkommensverteilung, die sich aus den Marktprozessen heraus ergeben, zu verringern. Das Ausmaß, in dem dies erfolgt, unterscheidet sich jedoch von Land zu Land deutlich. Auch die USA reduzieren die Ungleichheit der Markteinkommen, allerdings in einem viel geringeren Ausmaß als dies in Deutschland der Fall ist. Der GiniIndex der verfügbaren Einkommen lag in den USA in 2016 nur um 12,3 Punkte 


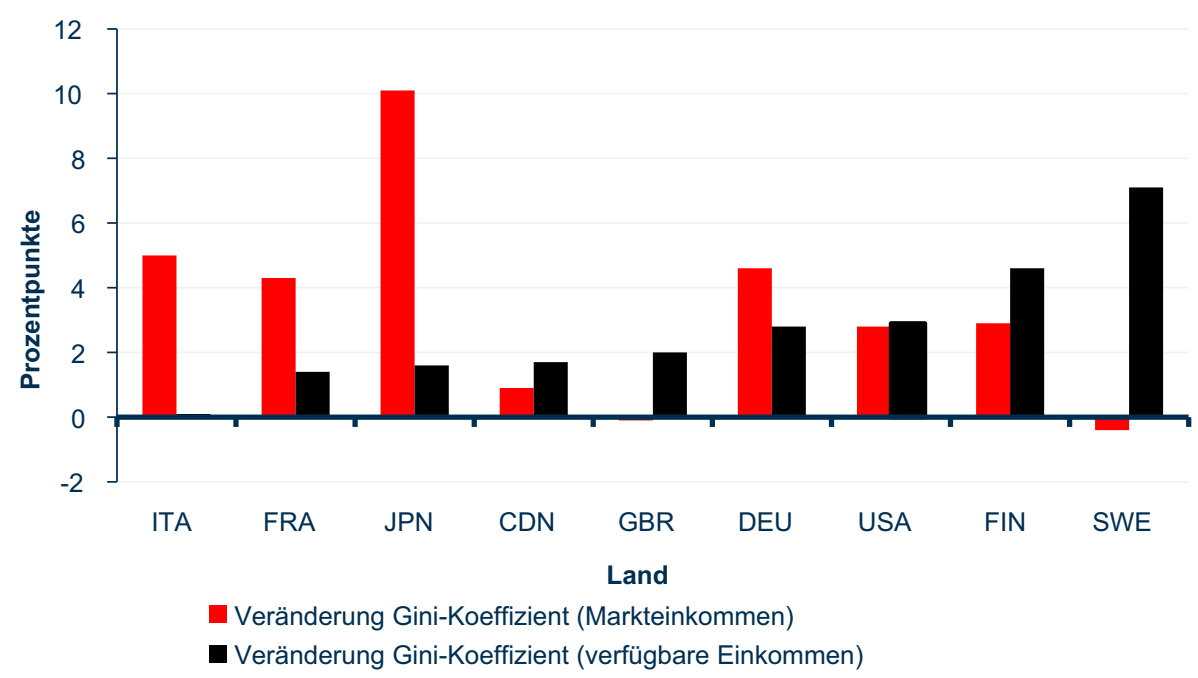

Abb. 2 Veränderungen der Gini-Koeffizienten der Einkommensverteilung in ausgewählten OECD-Ländern, 1995 bis ca. 2016 (Die Veränderung der Einkommensdefinition bei den Markteinkommen ab 2012 schränkt die Vergleichbarkeit ein. DEU, FRA, ITA: 1996-2016; JPN: 1995-2015; GBR: 1994-2017. Eigene Berechnungen. Gini-Koeffizient: $0=$ völlige Gleichverteilung, $100=$ völlige Ungleichverteilung der Einkommen). (Quelle: OECD 2020; Abruf am 05.01.2020)

unter dem Gini-Index der Markteinkommen. In Deutschland betrug die Differenz dagegen 21,2 Punkte.

Hierin zeigt sich, dass die Wirtschaftsordnung Deutschlands im Unterschied zu den USA als eine soziale Marktwirtschaft konzipiert ist, die darauf angelegt ist, soziale Härten durch Umverteilung und andere Formen des sozialen Ausgleichs abzufedern. Dies erfolgt sowohl durch eine monetäre Kompensation als auch durch die Zurverfügungstellung von öffentlichen Gütern. Aber nicht nur Deutschland betreibt eine nennenswerte staatliche Verteilungspolitik: auch Frankreich reduzierte durch Umverteilung die Differenz zwischen Markteinkommen und verfügbaren Einkommen um 21,2 Gini-Punkte - und damit in etwa in einem gleichen Umfang wie Deutschland. Wie Abb. 1 weiter verdeutlicht, verringerten Länder wie Irland oder Finnland die Ungleichheit durch ihre Umverteilungsmaßnahmen noch stärker als Deutschland und Frankreich. Auf der anderen Seite gibt es Länder wie Mexiko oder die Türkei, bei denen die Ungleichheit der verfügbaren Einkommen in 2015 kaum unter der der jeweiligen Markteinkommen lag. Bei der Ungleichheit der verfügbaren Einkommen liegt Deutschland im OECD-Länder-Vergleich im unteren Mittelfeld.

In Deutschland hat sich etwa zwischen Mitte der 1990er-Jahre und dem Jahr 2016 die Ungleichheit der Markteinkommen mit 4,5 Punkten auch im internationalen Vergleich deutlich erhöht (vgl. Abb. 2). Damit war die Zunahme in Deutschland sogar größer als in den USA (+3 Punkte). Anders als in Italien, wo eine starke Zunahme der Ungleichheit der Markteinkommen (+5,7 Punkte) nur zu einer geringfügig größeren Ungleichverteilung der verfügbaren Einkommen führte (+0,6 Punkte), hat sich der Gini-Index der verfügbaren Einkommen in Deutschland mit fast 2,7 Punkten ebenfalls spürbar erhöht. Der Fall Italien zeigt eindrücklich, dass eine wachsende 


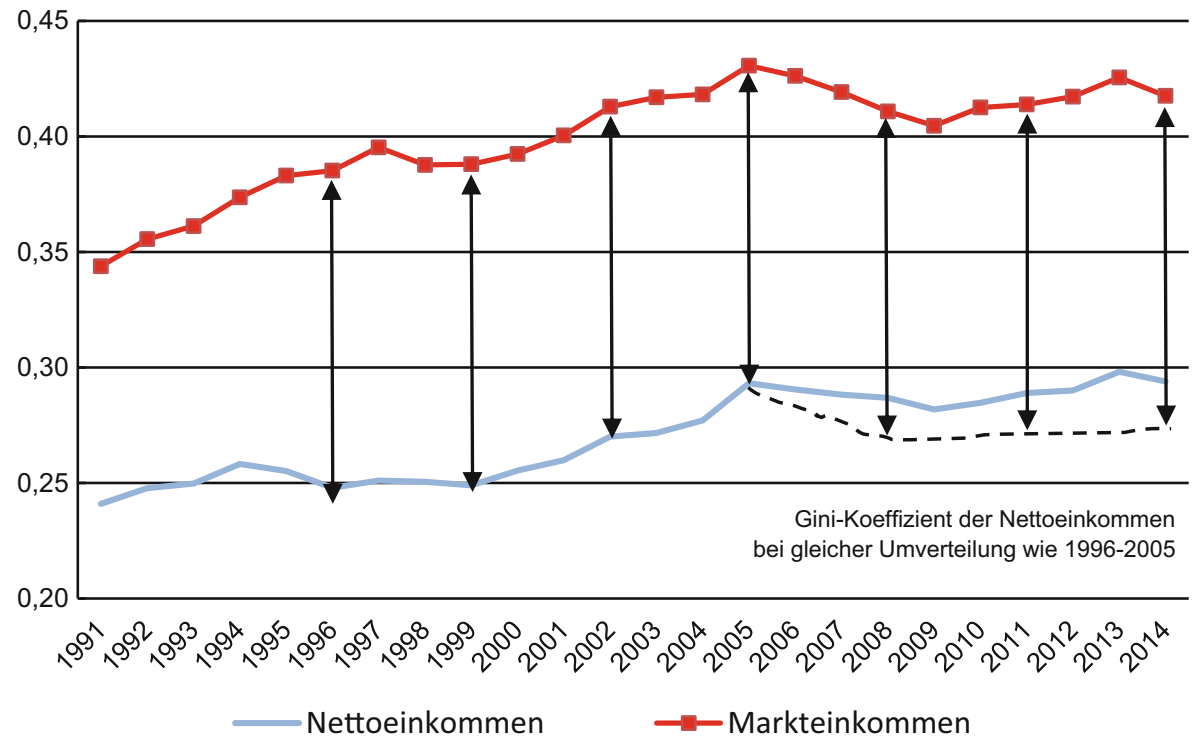

Abb. 3 Gini-Koeffizient der Markt- und Nettoeinkommen (äquivalenzgewichtet) in Deutschland, 1991-2014 (Markteinkommen: Einkommen vor Steuern und Transferleistungen. Nettoeinkommen: Einkommen nach Steuern und Transferleistungen. Für Personen in Haushalten mit mindestens einem erwerbsfähigen Mitglied. Gini-Koeffizient: $0=$ völlige Gleichverteilung, $1=$ völlige Ungleichverteilung der Einkommen. Ursprungsdaten: SOEP v 32 und Mikrozensus. Vgl. hierzu auch den Vortrag von G. Felbermayr auf dem IMK-Forum am 21.03.2018, online unter https://youtu.be/iXnz4-hFLrg sowie Felbermayr et al. (2016)). (Quelle: SVR 2017, S. 412)

Ungleichheit bei den Markteinkommen nicht zwangsläufig auch zu größerer Ungleichheit bei den verfügbaren Einkommen führen muss. Mit anderen Worten: die wachsende Ungleichheit der Markteinkommen, die sich in den vergangenen 25 Jahren ergeben hat, wurde in Deutschland nicht durch verstärkte Umverteilungsmaßnahmen kompensiert. Wenn man die am Gini-Index gemessene Ungleichheit hätte konstant halten wollen, wäre es notwendig gewesen, das Ausmaß der Umverteilung deutlich zu erhöhen. Da dies nicht der Fall war, sind in Deutschland heutzutage auch die verfügbaren Einkommen ungleicher verteilt als noch vor 25 Jahren.

Auch Abb. 3 zeigt, dass sowohl der Gini-Koeffizient der Markt- als auch der der Nettoeinkommen in Deutschland zwischen 1991 und 2014 gestiegen ist. Wenn man die Differenz zwischen dem Gini-Koeffizienten der Markteinkommen und dem Gini-Koeffizienten der Nettoeinkommen als Ausmaß der (monetären) Einkommensumverteilung interpretiert, dann geht aus dieser Abbildung außerdem hervor, dass die Umverteilung das beabsichtigte Ziel, dass die Nettoeinkommen weniger ungleich verteilt sind als die Markteinkommen, grundsätzlich erreicht hat. Ferner zeigt sich aber auch, dass sich das Ausmaß der Umverteilung in Deutschland in diesem Zeitraum verringert hat. Die gestrichelte Linie gibt den hypothetischen GiniKoeffizienten der Nettoeinkommen an, der sich ergeben hätte, wäre das Ausmaß der Umverteilung nach dem Jahr 2005 genauso hoch gewesen, wie im Zeitraum 1996-2005. 


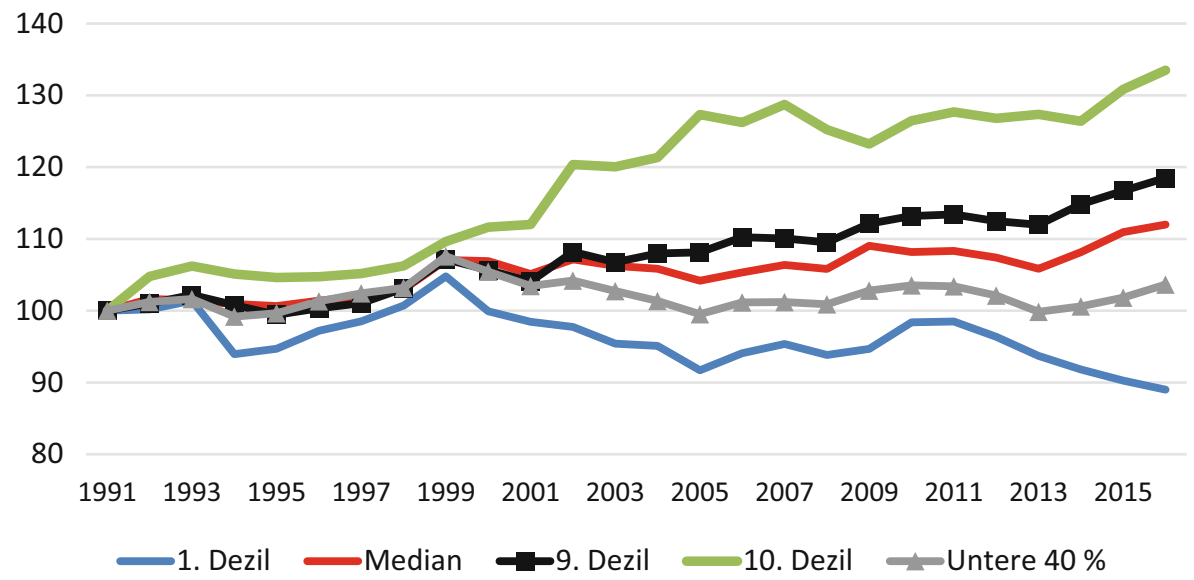

Abb. 4 Entwicklung der realen Nettoeinkommen nach Dezilen in Deutschland, 1991-2016 (Reale verfügbare Haushaltseinkommen (äquivalenzgewichtet) in Preisen von 2010. Für Personen in Privathaushalten mit mindestens einem erwerbsfähigen Mitglied. Datenbasis: SOEPv 33.1. 1991= 100. Daten zur Verfügung gestellt von Markus Grabka, DIW). (Quelle: SOEP)

\subsection{Verteilung des Nettoeinkommens nach Dezilen in Deutschland}

Der Gini-Koeffizient ist zwar das verbreitetste Maß der Einkommensverteilung vielleicht, weil er auf den ersten Blick leicht verständlich erscheint - allerdings weist diese Kennzahl aufgrund ihrer speziellen Konstruktion den bedeutsamen Nachteil auf, dass sie auf Veränderungen am oberen und unteren Ende der Einkommensverteilung relativ träge reagiert. Aber genau dort haben sich in der jüngeren Vergangenheit die stärksten Veränderungen ergeben. ${ }^{1}$

Daher sollen mit den folgenden Abbildungen noch Informationen über die Entwicklung der Verteilung, vor allem am unteren Rand, gegeben werden. Dazu werden Quantilsverteilungen betrachtet. Abb. 4 zeigt die Veränderung des realen Nettoeinkommens des Medians sowie des ersten und des neunten Dezils der Verteilung. Zwischen 1991 und 2014 ist das reale Median-Nettoeinkommen in Deutschland um knapp 10\% gestiegen. Das neunte Dezil verzeichnete eine fast doppelt so hohe Wachstumsrate wie der Median. Ganz anders die Situation für die untere Einkommensschicht: für das erste Dezil der Einkommensverteilung ergab sich im Betrachtungszeitraum ein Rückgang um rund $10 \%$.

Abb. 4 zeigt ferner die Entwicklung des realen Nettoeinkommens der unteren 40\% der Einkommensverteilung. Gegenüber 1991 hat diese Gruppe im Jahr 2016 einen recht bescheidenen Einkommenszuwachs von weniger als 4\% erzielen können. In diesem Zeitraum können zwei verschiedene Phasen unterschieden werden.

\footnotetext{
1 Aus diesem Grund lehnen Piketty und seine Kollegen, die gemeinsam die umfassende World Inequality Database (WID.world) aufgebaut haben, die Verwendung des Gini-Index ab; sie taucht in der Datenbank überhaupt nicht auf. Stattdessen verwenden sie Quantilsmaße (wie z. B. Dezile), mit denen die Einkommensanteile dargestellt werden, die auf bestimmte Gruppen in der Bevölkerung entfallen. Vgl. Alvaredo et al. (2020).
} 
Zunächst einmal stieg in der zweiten Hälfte der 1990er-Jahre das Nettoeinkommen der unteren $40 \%$ vergleichsweise kräftig an, um aber anschließend bis Mitte der 2000er-Jahre wieder deutlich zurückzugehen. Von 2005 bis 2016 wuchsen die realen Nettoeinkommen unter Schwankungen zwar wieder. Allerdings verfügten die unteren 40\% der Haushalte im Jahr 2016 immer noch über ein geringeres reales Nettoeinkommen als im Jahr 2000.

\subsection{Ursachen für die wachsende Ungleichheit der Einkommensverteilung}

Die Gründe für die wachsende Ungleichheit der Einkommen sind sowohl auf nationaler als auch auf internationaler Ebene vielfältig. ${ }^{2} \mathrm{Zu}$ den Faktoren, die die Einkommensverteilung haben ungleicher werden lassen, zählen unter anderem die Globalisierung und ein qualifikationsverzerrter technischer Fortschritt, die beide zu einer zunehmenden Spreizung der Lohnstruktur geführt haben. Auch die Erhöhung der Gewinnquote hat angesichts der Konzentration der Kapitaleinkommen bei den reicheren Haushalten bewirkt, dass die Einkommen ungleicher verteilt wurden (ILO et al. 2015). Veränderungen von institutionellen Rahmenbedingungen und ein Rückgang des gewerkschaftlichen Organisationsgrades haben ebenfalls eine wichtige Rolle gespielt (Freeman 2020). Beide machten es den Gewerkschaften schwerer, größere Einkommenssteigerungen durchzusetzen. Atkinson (2015, S. 4) hebt die Bedeutung der sogenannten Arbeitsmarktflexibilisierung hervor, die zu einer Machtverschiebung zu Ungunsten der Arbeitnehmer geführt habe.

Einen weiteren Beitrag zu dieser Entwicklung hat sicherlich auch die verminderte Umverteilung über das Steuer- und Transfersystem geleistet. Die Steuerpolitik hat sich in den letzten Jahrzehnten in vielen Ländern vor allem durch eine Reduzierung des Spitzensteuersatzes ausgezeichnet. Nach Ende des Zweiten Weltkrieges bis zur Mitte bzw. zum Ende der 1970er-Jahre wurden in den entwickelten Volkswirtschaften Spitzeneinkommen teilweise recht hoch besteuert. So betrug bis dahin beispielsweise der Spitzensteuersatz in Großbritannien deutlich mehr als 90\%, in den USA lag er bei $70 \%$. Später wurden auch in Frankreich und Deutschland die Spitzensteuersätze deutlich reduziert. Dies hat nicht nur einen Einfluss auf die Verteilung der Nettoeinkommen. Piketty (2014) hebt hervor, dass dies auch die Ungleichheit der Markteinkommen verändert habe, da die Spitzenverdiener bei sehr hohen marginalen Steuersätzen kaum einen Anreiz hätten, sich für eine Erhöhung ihrer Einkommen einzusetzen. Bei geringeren Spitzensteuersätzen verbleibt ihnen dagegen ein nennenswerter Anteil einer Einkommenserhöhung, so dass sich Verhandlungen über die Höhe von regulären Gehältern und erfolgsabhängigen Bonuszahlungen wieder lohnen. Auch in Deutschland haben die Reformen der Einkommens- und der Unternehmenssteuer der Schröder-Regierung um die Jahrtausendwende die Besserverdienenden teilweise erheblich entlastet. Gleichzeitig wurden bestimmte Transferausgaben für die schlechter gestellten Teile der Bevölkerung beschränkt (u. a. Hartz IV). Insgesamt wurde so die monetäre Umverteilung der Einkommen in Relation zum

\footnotetext{
2 Milanović (2016) bezeichnet die wichtigsten Determinanten einprägsam als die TOP-Faktoren - dabei steht T für Technological Progress, O für Openess, also die Effekte der Globalisierung, und P für Politics. Dieser Abschnitt basiert teilweise auf Hartwig und Krämer (2018).
} 
Nationaleinkommen reduziert. Wäre es ein Ziel gewesen, die Ungleichheit der Nettoeinkommen nicht größer werden zu lassen, hätte die Umverteilung angesichts einer steigenden Ungleichheit der Markteinkommen tatsächlich erhöht werden müssen.

Die Trends, die in den letzten 30-40 Jahren zu einem Anstieg der wirtschaftlichen Ungleichheit geführt haben, werden auf absehbare Zeit weiterhin wirksam sein. Eine Umkehr dieser Trends ist jedenfalls gegenwärtig nicht absehbar. Im Gegenteil: die fortschreitende Digitalisierung hat das Potenzial, die Polarisierung der Einkommen noch weiter zu erhöhen (Südekum 2018).

\section{3 Überlegungen zur distributiven Gerechtigkeit}

Wirtschaftliche Aktivitäten des Staates haben, ob beabsichtigt oder nicht, fast immer Auswirkungen auf die Einkommensverteilung. Daher sollten sich Ökonominnen und Ökonomen nicht nur mit Effizienzauswirkungen staatlichen Handelns, sondern auch mit Fragen nach der Gerechtigkeit der Einkommensverteilung beschäftigen.

\subsection{Ausgewählte Denker und Denkschulen: Prinzipien, Normen und Ziele der Einkommensverteilung}

Bereits für die Philosophen des antiken Griechenlands hatte das Problem der gerechten Verteilung einen hohen Stellenwert. ${ }^{3}$ Auch viele Denker des Mittelalters, die ökonomischen Frühklassiker und vor allem die Klassiker der politischen Ökonomie beschäftigten sich immer wieder mit der ,sozialen Frage“, die seinerzeit vor allem eine Frage nach den Ursachen des Reichtums von Wenigen bei gleichzeitiger Existenz von bitterster Armut von Vielen war. In den heute vorherrschenden wirtschaftswissenschaftlichen Theorien sucht man dagegen eine systematische Behandlung des Reichtums vergebens. Diese Thematik überlassen die meisten Ökonomen der Gegenwart den Soziologen oder den Philosophen. Ein Grund liegt darin, dass die Wirtschaftswissenschaft ihrem Selbstverständnis nach eine positive Wissenschaft ist, wodurch normative Verteilungsfragen als Untersuchungsobjekt aus der Ökonomik mehr oder weniger verschwunden sind. Zum Zweiten hat dies auch damit zu tun, dass das vorherrschende neoklassische Paradigma die „Verteilungsfrage“ - von einem theoretischen Standpunkt aus betrachtet - in einem vollkommenen Wettbewerbssystem als gelöst ansieht. Daher kann man sich zurecht die Fragen stellen, ob ,die ökonomische Theorie (insbesondere die Neoklassik) überhaupt in der Lage [ist], Verteilungsfragen angemessen zu würdigen“ oder sie sich ,mit ihrer starken Betonung der Allokationseffizienz selbst den Blick darauf [verstellt]?“ (Klodt 2018, S. 73). Dies war in der Geschichte der wirtschaftswissenschaftlichen Theorie nicht immer so. In der Klassischen Politischen Ökonomie standen Fragen nach dem Zusammenhang von Wachstum und Verteilung im Zentrum der Analyse. Doch nicht nur in der antiken Philosophie und in der ökonomischen Klassik war die Verteilungsfrage bedeutsam, auch in anderen wirtschaftswissenschaftlichen Paradigmen

\footnotetext{
3 Der folgende Abschnitt basiert in Teilen auf Krämer (2017).
} 
spielte sie zum Teil eine recht große Rolle, wie der folgende, überblickartig gestaltete Abschnitt zeigen soll.

\subsubsection{Platon}

Der griechische Philosoph Platon (427-347 v. Chr.) entwarf in den Nomoi (Die Gesetze), die Vision eines idealen Staates. Ethische Normen und die Gerechtigkeit der Verteilung spielen darin eine bedeutsame Rolle. Privateigentum und individuelles Vermögen sind in diesem Staatskonzept von Platon durchaus vorgesehen, aber der Philosoph missbilligt das Streben nach Reichtümern. Platon warnt eindringlich vor den Folgen zu großen Reichtums, da dieser seiner Meinung nach zu einem moralischen Verfall in der Gesellschaft führen würde. Zudem ginge Reichtum mit einer entsprechenden politischen Macht einher. Und unbeschränkte Macht würde zu Korruption und letztendlich in die Tyrannei führen (Kurz 2013, S. 14). Platon erkannte auch die soziale Sprengkraft, die aus der gleichzeitigen Existenz von tiefer Armut und übermäßigem Reichtum resultiert. Er fordert daher eine dementsprechende Begrenzung von Einkommen und Vermögen:

Es muß nämlich ... in einem Staate, welcher von Aufruhr und Spaltung frei bleiben soll ... weder der eine Teil der Bürger in drückender Armut, noch der andere in Reichtum leben, ... und so muß denn der Gesetzgeber nunmehr jedem von beiden eine Grenze setzen. (Platon 1862, 5. Buch)

Platon gibt sogar eine konkrete Grenze an, ab der er den Reichtum eines Einzelnen für zu groß hält und spricht sich bei deren Überschreiten für eine Umverteilung aus. So zeichnet sich für ihn eine gerechte Gesellschaft dadurch aus, dass der Besitz des Reichsten um nicht mehr als das Vierfache über den Besitz des Ärmsten hinausgeht. „[W]enn aber jemand noch mehr im Besitz hat, so soll er den Überschuss ... an den Schatz des Staates und seiner Schutzgötter abgeben“ (ebd.).

Eine einfache Übertragbarkeit dieser Verteilungsnorm auf die heutige Zeit verbietet sich jedoch nicht zuletzt dadurch, dass Platon die genannte Einkommensspanne nicht auf alle Gesellschaftsmitglieder bezog, sondern nur auf solche, die über Bürgerrechte verfügten. So wurden unter anderem Sklaven, die einen erheblichen Teil der gesellschaftlichen Arbeit erledigten, aber keinerlei Einkommen bezogen oder über nennenswerte Besitztümer verfügten, aus der Betrachtung ausgeschlossen. ${ }^{4}$

\subsubsection{Adam Smith}

Adam Smith (1723-1790) hat sich an vielen Stellen in seinen Schriften besorgt darüber gezeigt, wie elend die Lage der Arbeiter zu seiner Zeit gewesen ist. Sein wirtschaftspolitisches Credo zur Lösung der sozialen Frage lautete: Wirtschaftswachs-

\footnotetext{
4 Sen bemerkt dazu, dass die Wahrnehmung und der Sinngehalt von Ungerechtigkeit auch von den Möglichkeiten abhängen, sich tatsächlich dagegen auflehnen zu können. Daher fanden es die ,Intellektuellen im alten Athen [...] nicht besonders anstößig, bei ihren Diskussionen über Gleichheit die Sklaven außer Betracht zu lassen, und ein Grund dafür lag darin, daß sie sich das praktisch leisten konnten“ (Sen 2009, S. 19).
} 
tum! Nur durch eine marktwirtschaftliche, staatliche und gesellschaftliche Ordnung, die in der Lage sei, einen dynamischen Wachstumsprozess in Gang zu setzen, würde sich die Lage der arbeitenden Bevölkerung allmählich verbessern und langfristig die extreme Armut beseitigen lassen. Adam Smith (2012 [1776]) hatte gegenüber dem Reichtum Einzelner jedoch durchaus ein ambivalentes Verhältnis. Einerseits betrachtete er individuellen Reichtum skeptisch, soweit dieser zu Verschwendung, Habgier, Eitelkeit und ähnlichen Dingen verführte; hier sprach der Moralphilosoph aus ihm. Auf der anderen Seite hat Reichtum, soweit er sich in der Anhäufung von produktivem Kapital manifestiert, bei Smith und generell in der klassischen politischen Ökonomie bis hin zu Marx (1962-64 [1867-94]) eine zentrale ökonomische Funktion für die Wachstumsdynamik einer Ökonomie. Den wohlhabenden Kapitaleignern (nicht jedoch den unproduktiven Grundbesitzern!) kommt die zentrale Rolle zur Entfaltung der ökonomischen Dynamik zu. Entscheidend ist für Smith demzufolge der Zweck, den das Anhäufen von Vermögen und damit der Reichtum letztendlich hat. Die Kapitalisten haben in Smith' System daher eine wichtige gesellschaftliche Funktion zu erfüllen. Durch die Akkumulation von (Real-)Kapital entwickeln sie die produktive Basis des ökonomischen Systems weiter und schaffen auf diese Weise materiellen und gesellschaftlichen Fortschritt, der zum „Reichtum der Völker" führt. ${ }^{5}$ Damit erfüllen sie unbewusst und aus eigentlich anders gelagerten Motiven (Eigennutz, Profitstreben) einen Zweck zum Wohle aller. Reichtum wurde von Smith und den anderen Vertretern der klassischen politischen Ökonomie dann als positiv beurteilt, wenn seine Besitzer dadurch ihre potentiellen Fähigkeiten als dynamische Triebkräfte des ökonomischen Systems verwirklichen können. Dies ist dann der Fall, wenn Reichtum nicht für (unproduktiven) Konsum verausgabt, sondern gespart und akkumuliert wird. Denn für Smith, wie für die meisten Ökonomen seiner Epoche, war Ersparnis gleichzusetzen mit Investition.

\subsubsection{John Stuart Mill}

John Stuart Mill (1806-1873) sah - anders als vor ihm Adam Smith (2012 [1776]) und David Ricardo (1951-1973 [1817]) - ökonomische Gesetzmäßigkeiten ausschließlich in der Produktionssphäre am Werk (Spahn 2002; Kurz 2002). In den Vorbemerkungen zu seinen Principles of Political Economy stellte er fest, dass bei der Verteilung des Einkommens und der Vermögen vergleichbare Gesetze nicht existierten:

Anders als die Gesetze der Produktion sind die Gesetze der Verteilung teilweise

Menschenwerk: denn die Art und Weise, wie Vermögen in einem gegebenen Gesellschaftszustand verteilt ist, hängt von den hier bestehenden Statuten und Gewohnheiten ab. (Mill 2016 [1848], S. 28)

Mill zufolge kann die Verteilung durch gesellschaftliche Institutionen und Normen beeinflusst werden. Auf diese Weise ließen sich die existierenden sozialen

\footnotetext{
5 Diese Sichtweise wird heute auch als „Trickle-down-Effekt“ bezeichnet. Ob dieser Effekt in modernen Gesellschaften zwangsläufig auftritt, wird zunehmend infrage gestellt (vgl. Ostry et al. 2014; Voitchovsky 2009; Hartwig 2018).
} 
Spannungen mithilfe einer Verteilungspolitik befrieden. Allerdings sind der Beeinflussung der Distribution durch die Politik bestimmte Grenzen gesetzt.

Nach Mills Ansicht wird die Verteilung von Einkommen und Vermögen durch den politischen Einfluss und die Stärke der darum ringenden Gruppen bestimmt. John Stuart Mill hat mit dieser Sichtweise die Analyse der Einkommens- und Vermögensverteilung fundamental verändert, indem er sie letztendlich ,politisierte“. Die grundlegende Idee, die die gesamte klassische politische Ökonomie auszeichnet, nämlich, dass ein Freiheitsgrad in der Verteilung existiert, macht das klassische System offen für machttheoretische Ansätze in der Verteilung. John Stuart Mills Analyse hat dies offengelegt. Einflussreiche Vertreter des Marginalismus sind dieser Sichtweise später jedoch entschieden entgegengetreten.

John Stuart Mill war neben Jeremy Bentham auch einer der wichtigsten Vertreter der Lehre des Utilitarismus. Nach Ansicht der Utilitaristen müsse es das Ziel einer utilitaristischen Gesellschaftsordnung sein, ,,das größte Glück der größten Zahl“ zu gewährleisten. Um dieses zu erreichen, sei eine Verringerung von ökonomischer Ungleichheit notwendig. Denn aus utilitaristischer Sicht ist ein steigendes Einkommen mit einem sinkenden Grenznutzen verbunden. Jede zusätzliche Geldeinheit, um die sich das Einkommen eines Individuums erhöht, steigert dessen Nutzen in einem geringeren Ausmaß als die vorhergehende Geldeinheit. Daraus folgt, dass eine Geldeinheit, die von einem reichen zu einem armen Menschen umverteilt wird, den Gesamtnutzen in der Gesellschaft erhöht. Reichtum ist daher bei Mill - einem der Klassiker des wirtschaftlichen und politischen Liberalismus - im Unterschied zu seinen späteren liberalen Nachfolgern nicht sakrosankt. Konsequenterweise sprach sich John Stuart Mill nachdrücklich für substantielle Vermögens- und Erbschaftssteuern aus.

\subsubsection{Die utopischen Sozialisten und Marx}

Auch aus Sicht der Frühsozialisten war es nicht zu akzeptieren, dass einige Menschen Reichtümer anhäufen, während andere in Armut leben. Die utopischen Sozialisten gingen von der Prämisse der Gleichheit aller Menschen aus, was eine gleichmäßige Verteilung von Einkommen und Vermögen zur Konsequenz hat. Ähnlich sah es Karl Marx (1962-1964) [1867-1894], der aber im Unterschied zu Adam Smith aus den gesellschaftlichen Voraussetzungen und Auswirkungen des Akkumulationsund Entwicklungsprozesses im Kapitalismus ganz andere Schlussfolgerungen zog. Während Smith die Zustände der arbeitenden Klassen zwar durchaus bedauert, weist er den Arbeitern in seinem System jedoch keine aktive Rolle zu. Obschon ihre Arbeit die Quelle allen Reichtums sei, bringe erst die Tätigkeit des Kapitalbesitzers diese Eigenschaft hervor; dieser habe daher die Hauptrolle im Stück zu spielen. Die arbeitende Klasse ist bei Smith ökonomisch zwar größtenteils produktiv ${ }^{6}$, aber politisch zur Passivität verdammt. Aus der Arbeitswerttheorie, die die gesamte klas-

\footnotetext{
6 Adam Smith hat bekanntlich die Unterscheidung von produktiver und unproduktiver Arbeit in die ökonomische Theorie eingeführt und damit einen viele Generationen beschäftigenden „Produktivitätsstreit“ ausgelöst (Krämer 2019). Aber selbst die produktiven Arbeiter haben in der politischen Ökonomie von Smith eine untergeordnete Funktion.
} 
sische politische Ökonomie prägt, und seiner Geschichts- und Gesellschaftstheorie folgt bei Marx, dass die Arbeiter eines Tages der Aneignung des von ihnen geschaffenen Mehrwertes durch die Kapitalisten entgegentreten und durch eine Revolution eine klassenlose Gesellschaft ohne Privateigentum errichten werden (Kurz 2018).

\subsubsection{Neoklassik}

Mit dem Aufkommen der marginalistischen Schule (der sogenannten Neoklassik) in den 1870er-Jahren geriet die Frage nach den Prinzipien von Wachstum und Verteilung in den Hintergrund. Stattdessen wurde die Frage nach der Knappheit der Güter und Produktionsfaktoren zu der zentralen erkenntnisleitenden Untersuchungsfrage. Mit der Entstehung des Marginalismus verändern sich die Bestimmungsgründe von Wert und Preis und damit auch der Verteilung. Die sozialen Klassen verschwinden aus der Analyse und die Produktionsfaktoren Arbeit, Boden und Kapital treten an ihre Stelle. Die Einkommen dieser Produktionsfaktoren entsprechen im Marginalismus ihrem jeweiligen Wertgrenzprodukt, weshalb diese (im Wesentlichen mikroökonomische) Einkommensverteilungstheorie heute als „Grenzproduktivitätstheorie der Verteilung" bezeichnet wird. Unter bestimmten Annahmen über die Eigenschaften der Produktionsfunktion und bei vollkommenem Wettbewerb wird das Produkt vollständig und gemäß ihrem jeweiligen Wertbeitrag auf die beteiligten Produktionsfaktoren aufgeteilt. Alle Faktoreinkommen werden simultan bestimmt und entsprechen ihren jeweiligen wertmäßigen Grenzprodukten (Solow 1956).

Im Kern der mikroökonomischen Grenzproduktivitätstheorie geht es allerdings um eine Bestimmung der Nachfrage nach den einzelnen Produktionsfaktoren auf den jeweiligen Faktormärkten; im eigentlichen Sinne kann sie daher nicht als eine originäre Verteilungstheorie bezeichnet werden. Gleichzeitig mit der Bestimmung der Faktornachfrage werden auch die einzelnen Faktorpreise (Reallohn, Kapitalzins, Bodenrente) ermittelt. Anders als in der ökonomischen Klassik ist das Grundprinzip der Faktorpreisbestimmung für alle Produktionsfaktoren identisch.

Die Entlohnung der jeweiligen Produktionsfaktoren erfüllt das Kriterium der Leistungsgerechtigkeit. Ausbeutung kann es im Referenzfall der vollkommenen Konkurrenz nicht geben. Die Verteilung ist durch die Knappheit der Produktionsfaktoren bei gegebenen technischen Bedingungen der Produktion bestimmt und damit der sozialen bzw. politischen Auseinandersetzung entzogen. Raum für Konflikt und Verteilungsmacht besteht hier im Prinzip nicht.

Alfred Marshall (1842-1924) war der vielleicht wichtigste Protagonist, der mit seinen Principles (Marshall 1920) von 1890 der ökonomischen Revolution, also dem Paradigmenwechsel von der Klassik zum Marginalismus, zum Durchbruch verhalf (Streissler 1981, S. 41). Marshall war bestrebt, die ökonomische Wissenschaft nach John Stuart Mills Interventionen wieder zu „entpolitisieren“. Dies war auch ein Reflex auf die aufkommende Arbeiterbewegung und den Erfolg von sozialistischen und marxistischen Lehren. Das mit dem „ehernen Lohngesetz“ (Ferdinand Lasalle) beschriebene Grundprinzip von der quasi-naturgesetzlichen Bestimmung der Lohnhöhe, das Böhm-Bawerk (1914) in seinem bekannten Aufsatz über Macht oder ökonomisches Gesetz so vehement verteidigte, wurde im Rahmen der mikroökonomischen Produktionstheorie aufgenommen. Produktions- und Verteilungstheorie 
wurden dadurch zu zwei Seiten der gleichen Medaille. Allein die Gesetzmäßigkeiten des Marktes bestimmen die Verteilung des Produktionsergebnisses auf Kapital und Arbeit; die Distribution folgt damit quasi-naturgesetzlichen Prozessen. Da die außermarktliche Einflussnahme auf die Höhe des Lohnes oder anderer Verteilungsvariablen Kollateralschäden wie beispielsweise Arbeitslosigkeit hervorrufen werde, stelle sich die Frage nach „Gerechtigkeit“ einfach nicht. Wir werden weiter unten sehen, dass später insbesondere Friedrich August Hayek diese Auffassung dezidiert vertreten hat.

Allerdings wurde die Frage nach der Gerechtigkeit der Einkommensentstehung im Marktprozess und damit ebenso nach der Notwendigkeit von Umverteilung auch von neoklassischen Ökonomen immer wieder diskutiert. Diese kamen allerdings zu sehr unterschiedlichen Schlüssen: John Bates Clark (1847-1938) hielt beispielsweise einen Lohnsatz, der sich aufgrund der Marktprozesse ergibt, für quasi naturgesetzlich bestimmt und für grundsätzlich gerecht. ${ }^{7}$ Dagegen vertrat ein ,aufgeklärter Neoklassiker" wie der einflussreiche Paul A. Samuelson die Auffassung, dass der Markt allenfalls ein effizientes Ergebnis zustande bringen könnte, aber Verteilungsgerechtigkeit hieraus nicht abgeleitet werden könne (Kurz 2013).

Eine besondere Rolle innerhalb des marginalistischen Spektrums spielten Utilitaristen wie Edgeworth (1904) und Pigou (1912), die sich aus wohlfahrtstheoretischen Überlegungen für die Umverteilung von hohen Einkommen aussprachen. Grundlage ist das Prinzip, dass der Grenznutzen zunehmenden Einkommens immer weiter zurückgeht. Francis Y. Edgeworth (1845-1926) verstand sich dabei als Vertreter eines „exakten Utilitarismus“. Zwar sei der Nutzenvergleich zwischen zwei Individuen mit theoretischen Schwierigkeiten konfrontiert, dennoch sei dieser in der Praxis zulässig, wie Edgeworth meinte. Sofern die Individuen einigermaßen gleiche Präferenzen hätten, führe eine gleichmäßigere Einkommensverteilung zu einer größeren Wohlfahrt in der Gesellschaft. Eine zu ungleiche Einkommensverteilung verhindert nach dieser Denkweise „das größte Glück der größten Zahl““.

Arthur C. Pigou (1877-1959) befürwortete eine Umverteilung mit dem Ziel einer größeren Gleichheit und begründete dies ebenfalls mit dem Gesetz des abnehmenden Grenznutzens von steigendem Einkommen. Er schränkte die Sinnhaftigkeit der Umverteilung allerdings insoweit ein, als dass diese nicht dem von Alfred Marshall postulierten Prinzip der Wohlfahrtmaximierung zuwiderlaufen dürfe. Interessant ist Pigou auch deshalb, da er selbst in einem neoklassischen System, in dem prinzipiell die Grenzproduktivität die Faktoreinkommen bestimmt, die Möglichkeit von „Ausbeutung“ für möglich erachtet. Für Pigou kann die Grenzproduktivitätstheorie nämlich nur unter eng begrenzten Voraussetzungen zur Anwendung gelangen, die in der Realität nur selten vorlägen. In der realen Welt müssten Abweichungen von vollkommener Konkurrenz und damit Machteinflüsse nicht nur in wenigen Ausnahmefällen in Betracht gezogen werden. Je nach Größe der Preiselastizität des Angebots bzw. der Nachfrage sei es denkbar, dass ein Faktor nicht nach der Grenzproduktivität entlohnt werde. In diesem Fall liegt eine Pigou'sche Ausbeutung vor,

\footnotetext{
7, ,...] the distribution of income to society is controlled by a natural law, and [...] this law, if it worked without friction, would give to every agent of production the amount of wealth which that agent creates." (Clark 1927 [1899], S. v).
} 
die prinzipiell alle Faktoren betreffen kann. So kann beispielsweise auch das Kapital vom Arbeiter ,,ausgebeutet“" werden.

\subsubsection{Friedrich August Hayek}

Hayek erklärte den klassischen liberalen Vordenker John Stuart Mill für einen der Hauptschuldigen an der schieren Existenz der sogenannten Verteilungsfrage. Denn Mill, der wie oben dargestellt eine analytische Trennung von Produktions- und Distributionssphäre in die Wirtschaftstheorie eingeführt hatte, habe die aus Sicht Hayeks vollkommen falsche Vorstellung in die Welt gesetzt, dass es einen Spielraum bei der Verteilung geben könne:

Was wir heute erleben, ist das Ergebnis einer grundsätzlichen Konfusion, die von dem angeblich liberalen britischen Denker John Stuart Mill ausging. In seinen „Prinzipien der politischen Ökonomie“ schrieb Mill einen Satz, der die Grundlage für alle sozialistischen Ideen bildet: „Ist das Sozialprodukt erst einmal da, kann man damit machen, was man will.“ Dabei wurde völlig übersehen, dass der Produktionsprozess nicht unabhängig ist vom Verteilungsprozess, das heißt, man kann mit dem Sozialprodukt eben nicht machen, was man will. (Hayek 1981, S. 16)

Dass der Marktprozess Ungleichheiten hervorruft, war für Hayek offensichtlich. Diese Ungleichheiten seien aber keineswegs problematisch, sondern umgekehrt eine wichtige Voraussetzung für die wirtschaftliche Entwicklung, da sie eine zentrale Grundlage für die ökonomische Leistungsbereitschaft bilden würden:

Ungleichheit ist nicht bedauerlich, sondern höchst erfreulich. Sie ist einfach nötig. Leider Gottes ist das Sozialprodukt nur da, weil die Menschen nach ihrer Produktivität entlohnt und dorthin gelockt werden, wo sie am meisten leisten. Gerade die Unterschiede in der Entlohnung sind es, die den einzelnen dazu bringen, das zu tun, was das Sozialprodukt erst entstehen lässt. Durch Umverteilung lähmen wir diesen Signalapparat. (Hayek 1981, S. 16)

Den Begriff der „,sozialen Gerechtigkeit“ hielt Hayek für völlig deplatziert. Gerechtigkeit sei allenfalls als Kategorie des Rechtssystems sinnvoll, denn aus ihr könne man die Gleichheit der Individuen vor dem Gesetz ableiten. Sei diese nicht gewährleistet, wäre damit die Freiheit, das für Hayek höchste aller Güter (,die Voraussetzung aller anderen Werte“), zerstört. In der Wirtschaft dagegen habe der Begriff der Gerechtigkeit nichts zu suchen, da Hayeks Sichtweise zufolge Menschen auf den Märkten keinen Handlungsspielraum hätten und man deshalb ihre Handlungen auch gar nicht bewerten könne. Die Verteilung werde letztendlich allein von den Marktgesetzen bestimmt. Und hier hingen nach Hayek Produktion und Distribution untrennbar zusammen. Eine ungleiche Verteilung von Einkommen und Vermögen ist aus Sicht des Wirtschaftsliberalismus demnach ein gerechtfertigtes Resultat effizienter Marktprozesse. Hayek geht hier noch einen Schritt weiter, indem er die Frage nach der Gerechtigkeit der Einkommensverteilung in einer Marktwirtschaft als unsinnig ansieht und damit als unzulässig zurückweist, da: 
... die Verteilung, die aus dem Marktprozess hervorgeht, nicht das beabsichtigte Ergebnis menschlichen Handelns ist. Daher ist der Begriff der sozialen Gerechtigkeit in einer marktwirtschaftlichen Ordnung mit freier Berufswahl völlig sinnlos. (Hayek 1981, S. 16)

Hayek, der die Idee der sozialen Gerechtigkeit so verteufelt, setzt an dessen Stelle implizit ein anderes Konzept von Gerechtigkeit. Gerechtigkeit bedeutet für ihn nämlich ausschließlich Leistungsgerechtigkeit. Auf diese Weise kann ökonomische Ungleichheit wie folgt gedeutet und legitimiert werden: Reichtum ist Ausdruck vorangegangener Akkumulationsprozesse, die auf leistungsgerecht entlohnten Anstrengungen und Risiken basieren und die für die gesamte Gesellschaft positiv wirken. Dementsprechend warnt Hayek auch vor der Beschränkung von individuellem Reichtum:

Diejenigen, die die Reichen attackieren, vergessen, dass die meisten von ihnen im Verlaufe des Reichwerdens Arbeitsplätze schufen und so mehr Leuten geholfen haben, als wenn sie ihr Geld den Armen gegeben hätten. (Hayek 1981, S. 16)

Damit knüpft Hayek an den in unserem Zusammenhang zentralen Grundgedanken von Adam Smith an, wonach Kapitalakkumulation die zentrale Voraussetzung für Wirtschaftswachstum ist. Im Unterschied zu Hayek ist bei Smith eine Gleichsetzung von Reichtum mit Kapitalakkumulation und Wachstum aber keineswegs zulässig. Smith wies an vielen Stellen seines Werkes darauf hin, dass genauso die Möglichkeit bestünde, dass Reichtum für unproduktive Zwecke (Luxuskonsum, Geldhorte) verwendet wird. Hayek sieht dagegen offenbar selbst im verschwenderischen Luxusleben von Lebemännern eine gesellschaftlich nützliche Vorbild- und damit Entwicklungsfunktion. Indem diese einen Lebensstil verfolgten, bei dem neue Freizeitaktivitäten entstünden, könnte dies den Weg für die Entwicklung neuer Massenmärkte bereiten:

... many of the toys and tools of sport that later became the instruments of recreation of the masses were invented by playboys. (Hayek 2006 [1960], S. 195)

Bei Smith war jedoch weder der (verschwenderische) Konsum noch das eigennützige Verhalten eines Reichen eine Garantie für die Schaffung eines allgemeinen gesellschaftlichen Wohlstands. Erst die Einbettung in entsprechende gesellschaftliche Institutionen, die das eigennützliche Verhalten des Einzelnen in sinnvolle Bahnen lenkt, kann Smith zufolge dafür sorgen, dass aus Reichtum des Einzelnen auch der Reichtum des Volkes wird. Und außer Mill sahen auch andere liberale Klassiker des 19. Jahrhunderts gewisse Freiheitsgrade bei der Bestimmung der Verteilung. Hayeks Sichtweise geht somit an dieser Stelle über die Denktraditionen seiner liberalen Vordenker weit hinaus. Eingriffe des Staates zur Verringerung der aus dem Marktprozess resultierenden Ungleichheit der Einkommensverteilung lehnt Hayek sowohl auf der Ebene der Primär- als auch auf der der Sekundärverteilung strikt ab. Allenfalls zur Armutsreduktion dürfe der Staat in einem gewissen Umfang Einkommenstransfers durchführen oder öffentliche Güter bereitstellen. 
Ähnlich hat sich später auch Gregory Mankiw (2010, 2013) in der Diskussion über die Einkommen Top-1-Perzent geäußert. Mankiw kritisiert die politische Philosophie des Utilitarismus scharf und spricht sich gegen jegliche aus politischen Gründen motivierte Umverteilung von Einkommen aus. Er weist die Rawls'sche Idee eines institutionalisierten Systems der Einkommensumverteilung als einer Art Versicherung gegen die sozialen Risiken des Lebens zurück und stellt diesem eine sogenannte just-deserts-Perspektive entgegen. Aus dieser Perspektive gäbe es in einer Welt, in der nur vollkommene Wettbewerbsmärkte vorhanden sind und in der keine externen Effekte auftreten sowie keine öffentlichen Güter existieren, keinen vernünftigen Grund zur Erhebung von Steuern und zur Umverteilung von Einkommen. Denn Einkommen, die dem Wertbeitrag eines einzelnen Wirtschaftssubjekts entsprechen, seien im doppelten Wortsinn ,,verdient“ (just deserts) und damit gerecht. Nur bei allokativem Marktversagen, wie durch Abweichungen vom vollkommenen Wettbewerb und dem Auftreten von negativen Externalitäten, sei es notwendig und gerechtfertigt, (Pigou-)Steuern zu erheben. Auch zur Finanzierung öffentlicher Güter - zu denen er die Abwesenheit von Armut zählt - seien Steuern legitim. Ein progressives Steuersystem sei gemäß dem Äquivalenzprinzip zu gestalten und wäre nur dann gerecht, wenn diejenigen, die viel Steuern zahlen, auch überproportional von öffentlichen Gütern profitieren würden.

\subsection{Ansätze zur distributiven Gerechtigkeit}

Für Richard Musgrave (1957, 1959) war die „Distributionsabteilung“ neben der „Allokations- und Stabilisierungsabteilung“ ein integraler Bestandteil der Analyse der öffentlichen Finanzen. Musgrave et al. (1994, S. 113 ff.) entwickelten im Rahmen einer normativen Betrachtung des öffentlichen Sektors einige zentrale Ansätze zur Verteilungsgerechtigkeit, die im Folgenden aufgegriffen werden. Ihr Ziel war dabei, die Implikationen unterschiedlicher Gerechtigkeitsprinzipien zu verdeutlichen.

Die von Musgrave et al. (1994, S. $109 \mathrm{ff}$.) vorgestellten Kriterien für eine gerechte Einkommensverteilung weisen enge Bezüge $\mathrm{zu}$ den im vorhergehenden Abschnitt dargestellten unterschiedlichen Denkschulen und deren Auffassungen von einer gerechten Einkommensverteilung auf. Aufgeführt und diskutiert werden die folgenden Ansätze zur Verteilungsgerechtigkeit:

\subsubsection{Verschiedene Ansätze zur Verteilungsgerechtigkeit}

\section{Ausstattungskriterien}

a) jeder behält, was er am Markt verdient;

b) jeder behält, was er bei vollkommener Konkurrenz am Markt verdienen könnte;

c) jeder behält nur das Arbeitseinkommen;

d) jeder behält, was er auf einem vollkommenen Markt verdienen könnte, Startgleichheit vorausgesetzt. 


\section{Utilitarismus-Kriterien}

a) die Gesamtwohlfahrt wird maximiert;

b) die durchschnittliche Wohlfahrt wird maximiert.

\section{Gleichheitskriterien}

a) die Wohlfahrt aller wird angeglichen;

b) die Wohlfahrt der untersten Gruppe wird maximiert;

c) die kategoriale Gerechtigkeit erfordert die Bereitstellung von Sachgütern.

\section{Gemischte Kriterien}

a) es wird eine Wohlfahrtsuntergrenze festgesetzt, oberhalb derer das Ausstattungskriterium gilt;

b) die Verteilung wird so angepasst, dass die Wohlfahrt in Übereinstimmung mit gesellschaftlichen Wohlfahrtsgewichten maximiert wird (Quelle: Musgrave et al. 1994, S. 113 f.).

Vereinfacht gesprochen unterschieden sich diese Konzepte distributiver Gerechtigkeit darin, dass entweder die Individuen all das behalten dürfen, was sie selbst am Markt an Einkommen erzielen, so wie dies das Konzept der Ausstattungskriterien und damit das Prinzip der Leistungsgerechtigkeit vorsieht (Sen 2010). Oder es wird eine Maximierung der Wohlfahrt aller Gesellschaftsmitglieder angestrebt, wie das im Kern die utilitaristischen Kriterien vorschlagen. Eine wiederum andere Herangehensweise strebt die Gleichbehandlung aller Individuen an, die gemäß bestimmter Gleichheitskriterien umgesetzt wird. Betrachten wir diese Konzepte in der Darstellung von Musgrave et al. (1994, S. 113-121) noch etwas genauer:

1. Ausstattungskriterien Bereits Vertreter von naturrechtlichen Überlegungen, wie John Locke und Jean-Jacques Rousseau setzten das Recht eines Individuums auf die Früchte seiner Anstrengungen in den Mittelpunkt ihrer Gerechtigkeitsvorstellungen. Auf dieser Basis ist die Verteilung der Einkommen allein auf Grundlage der Faktorausstattung und deren Preisbildung am Markt legitimiert (Nozick 1974). Man kann sich dieses Prinzip in reiner Form vorstellen, wie dies das Ausstattungskriterium 1a vorsieht. Man könnte aber auch Einschränkungen der Art formulieren, wie dies in $1 b$ vorgenommen wird, wo nur Erträge, die auf einem idealen Wettbewerbsmarkt erzielt werden können, legitim sind. In dem Fall wären weder Monopolgewinne gerechtfertigt, noch Faktoreinkommen, die über das jeweilige Grenzprodukt hinausgehen. Eine weitergehende Möglichkeit in diesem Zusammenhang stellt Variante 1c dar, bei der das Ausstattungsprinzip nur für Arbeitseinkommen gilt, während Kapitaleinkünfte nicht legitim sind. Dieser Ansicht könnte die Vorstellung zugrunde liegen, dass Arbeit die einzige Quelle des Wohlstands ist und Kapitalerträge leistungsloses und damit ,unverdientes“ Einkommen (,unearned income“) sind. Schließlich fällt auch Variante 1d unter das Ausstattungskriterium. Hierbei werden die ungleichen Ausgangspositionen der Individuen, die realiter vorhanden sind, bei der Frage nach der Gerechtigkeit der Einkommensverteilung mitberücksichtigt. Demnach dürfte jeder nur das behalten, was er auf einem vollkommenen Markt 
verdienen könnte, sofern Startgleichheit gegeben wäre. In dem Fall würden Ungleichheiten als ungerechtfertigt angesehen, die aus Erbschaften, unterschiedlicher Ausbildung und einem unterschiedlichen Familienstand entstehen. Dagegen würden Ungleichheiten, die von angeborenen Unterschieden in der Erwerbsfähigkeit stammen, sowie das Resultat von unterschiedlichen Präferenzen zwischen Arbeit und Freizeit und eines verschiedenen Hangs zur Ersparnisbildung sind, als gerechtfertigt erscheinen.

2. Utilitarismus-Kriterien Die Vertreter des Utilitarismus lehnen es ab, angeborene Fähigkeiten sowie andere angeborene Ungleichheiten in der Ausstattung der Wirtschaftssubjekte als legitime Gründe für wirtschaftliche Ungleichheiten anzuerkennen. Sowohl der soziale Status als auch die Zufälligkeiten, die der „Gen-Lotterie" entspringen, sind ihrer Ansicht nach keine Legitimation für die Entstehung von wirtschaftlichen Ungleichheiten in jedwedem Wirtschaftssystem. Wie oben bereits ausgeführt, ist es Ziel der Utilitaristen, das Einkommen so zu verteilen, dass ,das größte Glück der größten Zahl“" erreicht wird. Hieraus folgt, dass das Einkommen auf alle Individuen gleichmäßig verteilt wird, sofern sie den gleichen Grenznutzen aus ihrem Einkommen beziehen. Bei ungleichen Grenznutzen kann es zu einer ungleichen Einkommensverteilung kommen, um zu erreichen, dass der Gesamtnutzen maximiert wird. In diesem Fall würden Individuen, die aus einem gleichen Einkommen einen höheren Grenznutzen beziehen, einen größeren Einkommensanteil erhalten.

3. Gleichheitskriterien Vertritt man die Auffassung, dass die Gleichheit der Individuen in einer Gesellschaft das oberste Ziel eines gerechten Wirtschaftssystems sein sollte, ist man vor das Problem der Operationalisierung gestellt. Was genau bedeutet Gleichheit? Daher lassen sich auch hier unterschiedliche Ausgestaltungen dieser Idee formulieren. Egalitäre Auffassungen, wie sie zum Beispiel Jean-Jacques Rousseau und Karl Marx vertreten haben, stellen auf die Gleichbehandlung aller Individuen ab (Position 3a). Dabei kann es allerdings nicht darauf hinauslaufen, die Einkommen vollkommen gleich auf alle Individuen oder Haushalte zu verteilen. Marx' bekannte Formulierung ,Jeder nach seinen Fähigkeiten, jedem nach seinen Bedürfnissen“" weist darauf hin, dass Faktoren wie die Größe der Familie, Beeinträchtigung der Gesundheit und ähnliche Dinge zu einem unterschiedlichen Bedarf führen. Egalität der Einkommensverteilung ist daher nicht notwendigerweise Ausdruck von Bedarfsgerechtigkeit. Sowohl die Anhänger des Utilitarismus als auch die des Egalitarismus muss das Problem der Effizienz von Umverteilungsmaßnahmen und die durch Steuern und Transfers verursachten Umverteilungsverluste beschäftigen. Wenn diese hinreichend groß sind, kann es passieren, dass sowohl diejenigen, die Einkommen abgeben, als auch diejenigen, die Einkommen empfangen, am Ende der Umverteilung schlechter gestellt sind als davor. Würde die Gleichheitsregel ins Extrem getrieben, kann es sein, dass am Ende eines solchen verteilungspolitischen Eingriffs alle gleich arm sind - ein Ergebnis, das nicht im Sinne der Vertreter des Gleichheitskriteriums sein kann.

Eine Abwandlung dieser Vorstellung, die in zentraler Weise auf das Problem der Armutsvermeidung abstellt, folgt den Ideen von John Rawls, der das Wohlergehen 
der Menschen nicht in einem utilitaristischen Sinn verstanden wissen wollte. Rawls schlug in seiner Theory of Justice (Rawls 1971) vor, dass Einkommensungleichheiten nur in dem Ausmaß reduziert werden sollten, wie sie dazu beitragen, die Einkommen für die Ärmsten der Gesellschaft zu verbessern (Kriterium 3b). Rawls ging von der Prämisse aus, dass die Zukunft von grundlegender Ungewissheit geprägt und kein einzelner Akteur in der Lage ist, vorherzusagen, welche Position er oder sie in ihr einnehmen wird. In einer solchen Situation, in der Entscheidungen hinter dem ,Schleier der Unwissenheit“ getroffen werden müssen, solle gemäß Rawls, das „Maximin-Prinzip“ gelten. Nach diesem Gerechtigkeitskriterium soll das soziale System so gestaltet werden, dass die Ausstattung derjenigen maximiert wird, die in der Gesellschaft am schlechtesten gestellt sind. Nach Rawls Vorstellungen kann das Verteilungsproblem durch die Bereitstellung von „Grundgütern“ angegangen werden.

Eine gewisse Modifikation hiervon stellt das Gleichheits-Kriterium 3c dar, das die kategoriale Gerechtigkeit anspricht. Hier wird die Minimalausstattung, über die Individuen zwecks Armutsvermeidung verfügen sollten, nicht in Form einer monetären Einkommensgröße, sondern im Hinblick auf die dazu notwenigen Konsumgüter definiert. Dazu gehört eine Minimalausstattung mit Nahrungsmitteln, Kleidung, Wohnraum usw. Diese Ausstattung kann dann entweder wieder in monetäre Einheiten umgerechnet oder in Form von öffentlichen Leistungen, wie öffentlich finanziertem Wohnraum oder Lebensmittelgutscheinen, Lebensmitteltafeln und Ähnlichem, zur Verfügung gestellt werden.

Amartya Sen hält den Vertretern der Ansätze von kategorialer Gerechtigkeit entgegen, dass nicht ausschlaggebend sei, wie gut eine Person mit Gütern versorgt ist. Nach Sens Ansatz der Verwirklichungschancen (2009 [1973], 1985, 1999) ist vielmehr entscheidend, was eine Person mit den Gütern, über die sie verfügt, erreichen kann. Sen versteht unter Verwirklichungschancen ,die Möglichkeiten oder umfassenden Fähigkeiten (capabilities) von Menschen, ein Leben führen zu können, für das sie sich mit guten Gründen entscheiden konnten, und das die Grundlagen der Selbstachtung nicht in Frage stellt“" (Sen 2000, S. 29). Güter haben bestimmte Eigenschaften, die in Abhängigkeit von der jeweiligen Person in Funktionen (functionings) umgesetzt werden können. Güter werden nicht an sich geschätzt, sondern für die Funktionen, die sie ermöglichen. Sen unterscheidet Zustände des ,Seins und Tuns“ (z.B. gut ernährt zu sein, eine Unterkunft zu haben), von Gütern, die zu ihrer Erreichung eingesetzt werden (Nahrungsmittel, Wohnung). Die Verfügung über Ressourcen (wie z.B. ein Fahrrad) ist eine Voraussetzung, aber der Wert einer Ressource hängt letztlich von der Fähigkeit des Einzelnen ab, sie in Funktionen (wie z. B. Radfahren) umwandeln zu können. Diese Fähigkeit wird von vielen Faktoren beeinflusst, unter anderem von der persönlichen Konstitution des Individuums (z. B. seiner Gesundheit), sozialen Normen, der öffentlichen Infrastruktur (hier z. B. der Straßenqualität). Es reicht mit anderen Worten nicht aus, die Menge an Gütern und Dienstleistungen zu betrachten, die einem Individuum zur Verfügung stehen; es muss auch geprüft werden, ob diese es ihm wirklich ermöglichen, seine eigenen Bedürfnisse zu befriedigen und sein Leben frei zu gestalten. Armut bedeutet in die- 
sem Sinne einen Mangel und Reichtum ein hohes Maß an Verwirklichungschancen (Sen 2010 [2009], S. 279 ff.). ${ }^{8}$

Sen legt Wert auf die Feststellung, dass Freiheit kein absolutes Konzept ist. Nach Ansicht von Sen können sich Grundwerte wie Freiheit unterhalb eines bestimmten Wohlfahrtsniveaus nicht entfalten. Somit stellt erst die Befähigung eines Individuums dessen faktische Freiheit dar, zwischen verschiedenen Funktionskombinationen wählen zu können. Diese Auswahlentscheidungen bezüglich alternativer Funktionen bedeutet, dass eine solche Person über positive Freiheiten verfügt. Das Güterbündel x zu konsumieren, wenn ein Individuum es aus einer Vielzahl von Möglichkeiten frei gewählt hat, ist mehr wert, als es zu konsumieren, wenn es keine Alternativen gibt. Der Freiheitsraum wird jedoch von der zur Verfügung stehenden Güterausstattung abhängen. Die realen Möglichkeiten unterscheiden sich daher sehr stark zwischen den einzelnen Menschen (Gaertner 2009, S. 364).

Entscheidend für Aspekte der Umverteilung ist, dass der Wert von Gütern nicht nur von individuellen Präferenzen, sondern viel mehr von den sozialen Umständen abhängt, in die die Menschen eingebettet sind. Verteilungsgerechtigkeit darf sich im Sinne des Befähigungsansatzes von Amartya Sen dementsprechend nicht daran ausrichten, wie gleichverteilt das Einkommen bzw. die Güter in einer Gesellschaft sind, da die Menschen unterschiedliche Bedürfnisse haben. Behinderte Menschen benötigen beispielsweise andere und womöglich auch mehr Güter, um befähigt zu sein, wie andere am gesellschaftlichen Leben teilzunehmen. Der Staat hat daher nach Sen die Aufgabe eines ,,aktivierenden Vermittlers“ (Kubon-Gilke 2013, S. 437). Zur Befähigung der Individuen spielt die Umverteilung von Einkommen eine wichtige Rolle, sie reicht aber nicht aus. Das Bildungs-, das Gesundheits- und das Sozialsystem tragen in erheblicher Weise dazu bei, dass den „Menschen alle ihre Potentiale zur Nutzung von Gütern und Dienstleistungen tatsächlich auch zur Verfügung stehen“ (ebd., S. 437-8). Gerechtigkeit ist in diesem Ansatz die Gleichheit der Fähigkeiten: Nicht Wohlfahrt oder Einkommen oder Reichtum müssen gleichgestellt und maximiert werden, sondern die Wahlfähigkeit der Menschen.

4. Gemischte Kriterien In der Praxis werden die vorgenannten Ansätze wohl nicht in reiner Form umgesetzt werden können. Die Existenz unterschiedlicher Gerechtigkeitskonzepte führt in demokratischen Gesellschaften im Rahmen des politischen Prozesses notwendigerweise zu Kompromissen, bei denen verschiedene Konzepte miteinander kombiniert werden. Kriterium 4a entspricht weitestgehend dem ,,justdeserts“-Ansatz von Gregory Mankiw, der im vorherigen Abschnitt dargestellt wurde. Hierbei dominiert der Ausstattungsansatz, der aber mit der Auffassung verbunden wird, dass niemand unter Armut leiden solle..$^{9}$ Musgrave et al. (1994, S. 121) summieren:

\footnotetext{
8 Die praktische Bedeutung des Konzepts der Verwirklichungschancen von Amartya Sen zeigt sich auch daran, dass es seit 2005 eine konzeptionelle Basis für die Erarbeitung des deutschen Armuts- und Reichtumsberichts darstellt (Deutscher Bundestag 2005; Arndt et al. 2006).

9 Zur Erinnerung: Mankiw lehnt jegliche Steuer ab, die zur Umverteilung von Einkommen gedacht ist. Die Abwesenheit von Armut stellt für ihn aber ein öffentliches Gut dar. Da Mankiw die Erhebung von Steuern zur Finanzierung von öffentlichen Gütern als legitim ansieht, darf der Staat nach Mankiw Einkommenstransfers ausschließlich zur Armutsvermeidung vornehmen.
} 
Ein solcher Kompromiss (vielleicht kombiniert mit einer gewissen Berücksichtigung des Startgleichheitsprinzips im Rahmen der Ausstattungskriterien) kommt den gegenwärtigen Vorstellungen einer gerechten Verteilung wohl am nächsten. Anders ausgedrückt, es wird als gerecht angesehen, dass der Ausstattungsansatz im Hinblick auf die Erträge am Markt Gültigkeit haben sollte, aber das sich ergebende Ausmaß von Ungleichheit begrenzt werden sollte durch Setzen einer Untergrenze im Hinblick auf den Anteil, der am unteren Ende der Skala erreicht wird.

Als Richard und Peggy Musgrave diese Zeilen für die erste Auflage ihres Buches im Jahr 1973 verfassten, befand sich die ökonomische Ungleichheit in fast allen fortgeschrittenen Ländern auf einem vergleichsweise geringen Niveau. Mit dem „Inequality Turn“ (Atkinson), der sich zu Beginn der 1980er-Jahre ereignet hat, ist die ökonomische Ungleichheit jedoch wieder deutlich größer geworden - in einigen Ländern stärker als in anderen. Insofern ist das gemischte Kriterium 4b, bei dem die Verteilung so angepasst werden sollte, dass die Wohlfahrt in Übereinstimmung mit gesellschaftlichen Wohlfahrtsgewichten maximiert wird, heutzutage hinsichtlich der Auswahl eines geeigneten Maßstabs für distributive Gerechtigkeit vielleicht eher konsensfähig.

\subsection{Verteilungsnormen und Verteilungstypologien}

Eingangs wurde bereits darauf hingewiesen, dass bei der Behandlung von Verteilungsfragen normative Betrachtungen unumgänglich sind und dass sich die seit den 1870er-Jahren vorherrschende Ökonomik weitgehend von Werturteilen fernhält. ${ }^{10}$ Allerdings sind sich die Ökonomen auch auf dem Feld der positiven Theorie uneinig, welche wirtschaftlichen und sozialen Folgen eine ungleiche Verteilung hat und ob sie gegebenenfalls durch staatliche Eingriffe weitgehend problemlos zu korrigieren wäre. Wir haben im letzten Abschnitt gezeigt, dass auf der einen Seite eine ungleiche Verteilung der Markteinkommen - zumindest bis zu einem gewissen, schwer zu bestimmenden Ausmaß - als durchaus wünschenswert erachtet wird, wofür vor allem Anreizeffekte als Argument angeführt werden. Vertreter dieses Gedankens gehen davon aus, dass sich eine realiter ungleich verteilte Leistungsfähigkeit auch in korrespondierendem Einkommen niederschlagen müsse, um entsprechende Motive für ökonomische Aktivitäten zu gewährleisten. Ungleichheit der Einkommensverteilung, so der Grundgedanke, ist eine notwendige Voraussetzung dafür, dass die Effizienz eines marktwirtschaftlichen Systems maximal werden kann. ${ }^{11}$

Auf der anderen Seite werden aber auch zahlreiche negative Auswirkungen einer ungleichen Verteilung von Einkommen (und Vermögen) diskutiert. Hierzu gehören neben den klassischen Wohlfahrtsargumenten (Pigou 1920) auch makroökonomische

\footnotetext{
10 Sohmen (1976, S. 1) weist zurecht daraufhin, dass auch im Rahmen der Allokationstheorie Werturteile getroffen werden müssen. Wenn es in der Ökonomik um die optimale Allokation von Ressourcen gehe, dann kann , die Aufstellung von Bedingungen für ein gesamtwirtschaftliches Optimum niemals ,wertfrei sein“. Daher gehört „die Allokationstheorie zum Bereich der ,normativen“ Ökonomik“ (ebd.).

11 Der folgende Abschnitt basiert zum Teil auf Krämer (2014).
} 
Stabilitätsprobleme (z.B. Kumhof und Ranciere 2010) und negative Wachstumseffekte (z. B. Persson und Tabellini 1994). ${ }^{12}$ Auch den Vertretern dieser Argumentationslinie geht es dabei im Wesentlichen um Markteffizienz. Ihrer Auffassung zufolge hat Ungleichheit (ab einer bestimmten Höhe) einen negativen Einfluss auf die Allokationsfähigkeit eines Wettbewerbsmarktes. In diesem Sinn liegt ein allokatives Marktversagen vor, das Eingriffe des Staates in die Preisbildung auf dem Markt oder eine Umverteilung der Markteinkommen legitimiert, um eine gleichmäßigere Verteilung der Nettoeinkommen zu erreichen und damit die Effizienz des Systems zu erhöhen.

Wie nun jedoch beispielsweise von Fehr und Schmidt (1999) experimentell festgestellt wurde, ist Fairness offenkundig ein wichtiges Kriterium, das die Wahlhandlungen von Individuen beeinflusst. ${ }^{13}$ Die Frage nach der Gerechtigkeit einer gegebenen Einkommensverteilung gehört dazu. Dabei spielt die Einschätzung, wie die Höhe des eigenen Einkommens im Vergleich zu anderen ist, genauso eine Rolle, wie die Auffassung darüber, ob die allgemeine Einkommensverteilung der Gesellschaft als fair oder unfair anzusehen ist. Anders als im Grundmodell des Homo Oeconomicus unterstellt, beurteilen Individuen ihre Lebenszufriedenheit nämlich auch über Einkommensvergleiche mit anderen Individuen bzw. Referenzgruppen. Wie die experimentelle Ökonomik zudem gezeigt hat, existiert eine weit verbreitete Aversion gegen Verteilungen, die als ungerecht erachtet werden. Dies kann ökonomische Akteure dazu veranlassen, sich gegen eine unfaire Situation aufzulehnen (Carlsson et al. 2005). Außerdem hat eine egalitäre Einkommensverteilung einen positiven Einfluss auf zahlreiche soziale Indikatoren (Wilkinson und Pickett 2009) und die allgemeine Lebenszufriedenheit (Easterlin 1973, 1995). Eine gerechte Einkommensverteilung gehört damit gewissermaßen zur Zielfunktion einer Gesellschaft.

Das Ergebnis eines Marktprozesses, der sich auf einem vollkommenen Wettbewerbsmarkt vollzogen hat und daher dem Effizienzkriterium genügt, der aber in einer Einkommensverteilung resultiert, die keine Akzeptanz in der Gesellschaft findet, soll im Folgenden als normatives Marktversagen bezeichnet werden (Musgrave 1957, 1959; Gawel 2009). Unter solchen Voraussetzungen kann eine Korrektur der Einkommensverteilung sinnvoll sein und die gesellschaftliche Wohlfahrt steigern, selbst wenn dadurch die allokative Effizienz gemindert und das Sozialprodukt verringert wird.

Wenn aufgrund eines normativen Marktversagens die Einkommensverteilung durch staatliche Eingriffe korrigiert werden soll, muss eine Verteilungsnorm existieren, an der sich die Umverteilung orientieren kann. Einen gesellschaftlichen Konsens über eine wünschenswerte Verteilung zu erreichen, ist kein einfaches Unterfangen. Es ist sicher eher möglich, eine weitgehende Übereinstimmung darüber zu erzielen, dass eine gegebene Einkommensverteilung unangemessen ist. Betrachten wir stellvertretend für viele mögliche Kriterien einer gerechten Einkommensverteilung zunächst nur den Abstand zwischen den höchsten und den niedrigsten Einkommen. Dann lässt sich beispielhaft darauf verweisen, dass die in den USA herrschende Einkommensrelation vom 360-fachen der Vorstandsvergütungen im Verhältnis zu

12 Vgl. zum Zusammenhang von Wachstum und Verteilung auch Hartwig (2018).

13 Helmedag (2010) befasst sich ausführlich mit Aspekten von Fairness in der Lohnbildung. 
Tab. 1 Verteilungstypologien

\begin{tabular}{|c|c|c|}
\hline Verteilungstyp & $\begin{array}{l}\text { Verhältnis D10-Referenzwert }(=18 \%) \mathrm{zu} \\
\text { D10 im jeweiligen Verteilungstyp }\end{array}$ & $\begin{array}{l}\text { Verteilungsindex } \\
\text { (VI) }\end{array}$ \\
\hline $\begin{array}{l}\text { 1. Utopia } \\
\text { (Perfekte Gleichverteilung) }\end{array}$ & $18 \% / 10 \%$ & 1,8 \\
\hline $\begin{array}{l}\text { 2. Korporatistische Marktwirtschaft } \\
\text { (Degressive Verteilung) }\end{array}$ & $\begin{array}{l}18 \% / 10 \% \text { bis } \\
18 \% / 18 \%\end{array}$ & $1,8>\mathrm{VI}>1,0$ \\
\hline $\begin{array}{l}\text { 3. Soziale Marktwirtschaft } \\
\text { (Lineare Verteilung) }\end{array}$ & $18 \% / 18 \%$ & 1,0 \\
\hline $\begin{array}{l}\text { 4. Kapitalistische Marktwirtschaft } \\
\text { (Progressive Verteilung) }\end{array}$ & $\begin{array}{l}18 \% / 18 \% \text { bis } \\
18 \% / 100 \%\end{array}$ & $1,0>\mathrm{VI}>0,18$ \\
\hline $\begin{array}{l}\text { 5. Superstar-Kapitalismus } \\
\text { (Perfekte Ungleichverteilung) }\end{array}$ & $18 \% / 100 \%$ & 0,18 \\
\hline
\end{tabular}

den einfachen Mitarbeitergehältern Umfragen zufolge von einer großen Mehrheit als nicht angemessen empfunden wird. Viel schwieriger ist es dagegen, einen Konsens darüber herbeizuführen, welche Einkommensverteilung als fair anzusehen und daher anzustreben ist. Denn welche Relation sollte stattdessen gelten? Wäre das 100fache oder nur das Doppelte eine faire Verhältniszahl?

Da sich in der jüngeren Vergangenheit eine umfassende gesellschaftliche Debatte nach der Angemessenheit der Vergütungen der Topmanager in Relation zu anderen Erwerbseinkommen entzündet hat, soll im Folgenden die Frage nach einer angemessenen und fairen Spannweite zwischen den reichsten und ärmsten Einkommensbeziehern, die bereits von Platon als Maßstab einer gerechten Verteilung herangezogen wurde, in den Mittelpunkt rücken. ${ }^{14}$ Um ein umfassendes Bild einer fairen Verteilungssituation zu erhalten, soll für die weiteren Überlegungen die Sekundärverteilung betrachtet werden, die mittels der realen Haushaltsjahresnettoäquivalenzeinkommen dargestellt wird. Zudem soll die Einkommensverteilung anhand der Dezilsdarstellung analysiert werden, bei der die Haushalte nach der Höhe ihres Nettoeinkommens sortiert und in zehn gleich große Gruppen aufgeteilt werden. Das unterste (oberste) Dezil gibt dabei die Einkommenssituation der ärmsten (reichsten) zehn Prozent der Haushalte an.

Ein Blick auf empirische Verteilungswerte für das oberste Einkommensdezil in den USA und Deutschland offenbart das Ausmaß der Ungleichheit der Sekundärverteilung. Im Jahr 2014 flossen in den USA etwa 39\% und in Deutschland rund 30\% der Nettoeinkommen an das oberste Dezil der Haushalte. ${ }^{15}$ Um fundierter darüber urteilen zu können, ob dieser Anteil zu hoch, angemessen oder zu gering ist, benötigt man eine geeignete Referenzgröße. Als Maßstab der im Weiteren diskutierten Verteilungsnorm wird hierbei der Anteilswert des obersten Dezils herangezogen. In Abhängigkeit von der Höhe des Einkommensanteils, der an die oberen $10 \%$ der Haushalte im Verhältnis zu den anderen Haushalten fließt, sollen in einem ersten

\footnotetext{
14 Vgl. Abschn. 4.1 im vorliegenden Beitrag, in dem festgehalten wurde, dass für Platon eine gerechte Gesellschaft eine solche wäre, in der der Reichste nicht mehr als das Vierfache des Ärmsten besäße.

15 WID.World (Abruf der Daten 01.03.2020). Im Unterschied zu den USA, für die die Daten aktuell nur bis 2014 reichen, weist die Datenbank für Deutschland Werte bis ins Jahr 2016 aus. Der Anteilswert des obersten Dezils lag in Deutschland im Jahr 2016 in etwa in der gleichen Höhe wie im Jahr 2014.
} 
Schritt verschiedene Verteilungstypen definiert und einer davon als Referenz-Verteilungstyp ausgewählt werden. In einem zweiten Schritt werden dann zur Entwicklung einer umfassenden Verteilungsnorm noch zwei weitere Bedingungen hinzugefügt. In Tab. 1 sind fünf modelltypische Verteilungen aufgeführt:

1. Im Modell „Utopia“ erhält jeder Haushalt den gleichen Einkommensanteil. Jedem Dezil fließen demnach genau $10 \%$ des Gesamteinkommens zu.

2. Beim Typus „Korporatistische Marktwirtschaft“ sind die Einkommen je nach Dezil zwar unterschiedlich hoch, aber noch einigermaßen gleichmäßig verteilt. Vom ersten Dezil mit den ärmsten Haushalten (D1) bis zum letzten Dezil mit den reichsten Haushalten (D10) weist die Erhöhung der jeweiligen Einkommensanteile einen degressiven Verlauf auf.

3. Im Verteilungsmodell der „Sozialen Marktwirtschaft“ verdienen die Haushaltsgruppen ebenfalls unterschiedlich viel, aber die Verteilungsunterschiede sind größer als in Modell 2. Damit die Abstände zwischen den Dezilen nicht über alle Maße wachsen, wird angenommen, dass die absoluten Unterschiede im Einkommen zwischen den Dezilen jeweils gleich groß sind. Bei einer solchen linearen Verteilung hat beispielsweise das erste Dezil ein Haushaltseinkommen von 100 Einkommenseinheiten. Das zweite Dezil und jedes weitere Dezil haben ein Haushaltseinkommen, das 100 Einheiten höher ist, als das des vorhergehenden Dezils. Unter dieser Voraussetzung bekommt das einkommensstärkste Zehntel aller Haushalte etwa $18 \%$ des Gesamteinkommens. Dem ersten Dezil würden entsprechend rund $1,8 \%$ des gesamten Einkommens zufließen.

4. Im Modell der „Kapitalistischen Marktwirtschaft“ nehmen die Anteile vom ersten bis zum zehnten Dezil jeweils überproportional zu, demnach sind die Einkommen progressiv verteilt. In diesem Fall wird das zehnte Dezil mehr als $18 \%$ des Einkommens erhalten; dies korrespondiert mit dem realiter existierenden Anteil am gesamten Nettoeinkommen, das derzeit das zehnte Dezil in den USA bzw. in Deutschland erhält.

5. Beim fünften Modell „Superstar-Kapitalismus“ fließt das Gesamteinkommen ausschließlich an die obersten $10 \%$ der Haushalte, die anderen Haushalte erhalten nichts.

Wie sind die einzelnen Verteilungstypen nun unter dem Aspekt einer gerechten Einkommensverteilung zu beurteilen?

1. Das Modell 1 „Utopia“ mit einer vollkommenen Gleichverteilung der Einkommen widerspricht zentralen Gerechtigkeitskonzepten. Zum einen ist hier keine Bedarfsgerechtigkeit gegeben, da zwar die Haushaltsgröße und -zusammensetzung, aber nicht Faktoren wie Krankheit, Alter usw. berücksichtigt werden, die den Einkommensbedarf ebenfalls bestimmen. Zum anderen aber widerspricht es der Idee der Leistungsgerechtigkeit und dürfte folgenreiche negative Anreizeffekte auslösen.

2. Das Modell 2 ,Korporatistische Marktwirtschaft“" weist eine nur wenig von der perfekten Gleichverteilung abweichende Dezilsverteilung auf. Auch bei einer solchen Verteilung könnten sich die hierdurch verursachten negativen Anreizeffekte als recht kritisch erweisen. 
3. Das Verteilungsmodell 3 ,Soziale Marktwirtschaft“" weist eine zwar durchaus ungleichmäßige, aber noch keine extreme Ungleichverteilung der Einkommen auf. Man könnte diesen Verteilungstypus als einigermaßen akzeptablen Kompromiss zwischen Markteffizienz und Verteilungsgerechtigkeit verstehen. Somit bietet sich dieser Typus als Referenzverteilung an.

4. Im Verteilungsmodell 4 „Kapitalistische Marktwirtschaft“ sind die Einkommen sehr ungleich verteilt. Die hier bestehende progressive Verteilung zeichnet sich überdies dadurch aus, dass die Abstände nach oben immer größer werden. Diese Art der Verteilung herrscht gegenwärtig in den meisten OECD-Ländern vor.

5. Das Verteilungsmodell 5 ,Superstar-Kapitalismus“ stellt analog zum Modell 1 den anderen Extremfall dar; die Einkommen sind hier maximal ungleich verteilt. Eine solche Verteilung ist unter Gerechtigkeitsaspekten nicht vertretbar und aufgrund der prinzipiellen Funktionsunfähigkeit einer derartigen Ökonomie auch nicht möglich.

In Deutschland hat die Soziale Marktwirtschaft eine große Akzeptanz. Nimmt man grob vereinfachend an, dass eine lineare Verteilung einer anzustrebenden Verteilung im Rahmen einer sozialen Marktwirtschaft entspricht, lässt sich hieraus eine Verteilungsnorm für die Dezilstruktur der personellen Nettoeinkommen ableiten. Dementsprechend wird als Referenzwert ein Dezils-Verteilungsindex (DVI) definiert, bei dem der Anteil des zehnten Dezils bei einer linearen Einkommensverteilung $(\sim 18 \%)$ ins Verhältnis zum realiter vorliegenden Anteil des zehnten Dezils gesetzt wird. Im oben beschriebenen Sinne liegt eine anzustrebende Verteilung der Nettoeinkommen dann beim Wert Eins. Je weiter der Verteilungsindex nach oben abweicht, desto gleichmäßiger die Verteilung, aber umso kritischer das Anreizproblem. Je weiter der Verteilungsindex von Eins nach unten abweicht, desto größer die Leistungsanreize, aber umso mehr wird die soziale Gerechtigkeit beeinträchtigt.

Allerdings erfasst auch ein Verteilungsindex von Eins noch nicht alle Dimensionen einer ,idealen Einkommensverteilung“. Das Modell soll daher noch weiter spezifiziert werden, denn es ist durchaus möglich, dass das Einkommen des ersten Dezils eine absolute Höhe annimmt, die unterhalb des Existenzminimums liegt. ${ }^{16}$ Daher ist es sinnvoll, die Einkommenshöhe des ersten Dezils mit der Bedingung zu verknüpfen, dass damit eine Grundsicherung abgedeckt wird. Zum Beispiel dürfte dann kein Einkommensbezieher des ersten Dezils über ein Einkommen verfügen, das unterhalb der offiziellen EU-Armutsgefährdungsgrenze (dies sind $60 \%$ des medianen Äquivalenzeinkommens) liegt. Diese Überlegung lehnt sich an das „Quantilsaxiom“ von Basu (2011) an, der als normativen Orientierungspunkt für die Verteilungspolitik im Sinne von Rawls Maximin-Kriterium die Steigerung des Wohlergehens des ärmsten Fünftels der Bevölkerung vorschlägt. Da wir jedoch Dezile betrachten, wird dieses Kriterium nicht auf das ärmste Fünftel, sondern auf das ärmste Zehntel der Einkommensverteilung angewandt.

Will man auch das Ausmaß der Spreizung zwischen den reichsten und ärmsten Haushalten in die Verteilungsnorm integrieren, so kommt eine weitere Bedingung

16 Diese Überlegung lehnt sich an das von Musgrave et al. (1994) definierte gemischte Kriterium 4b an, wie es in Abschnitt 4.2 vorgestellt wurde. 


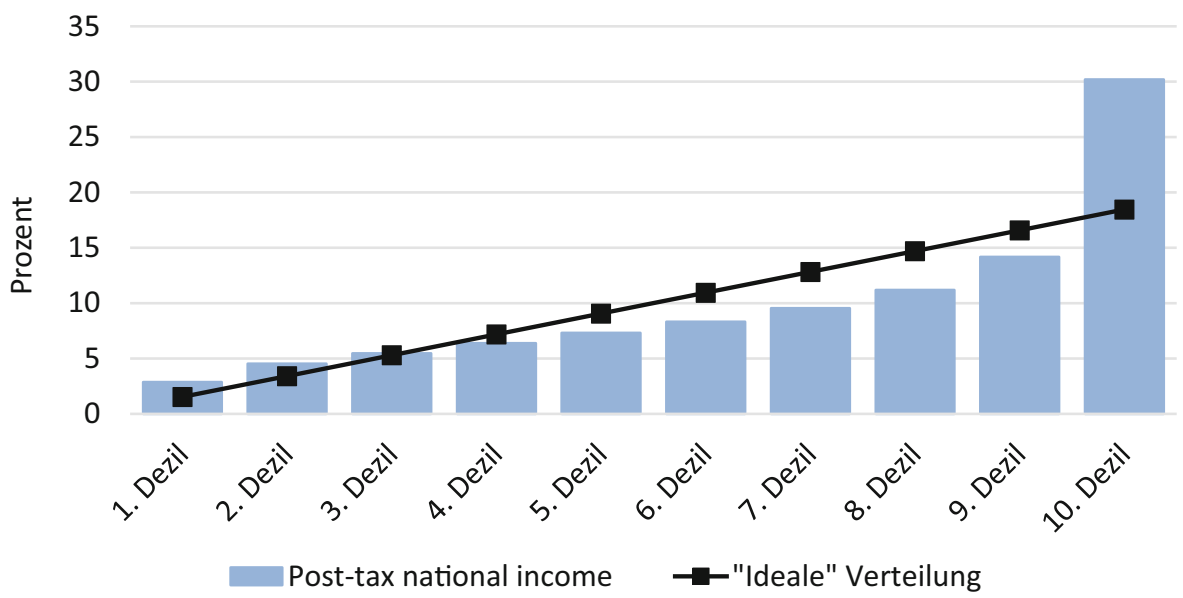

Abb. 5 Nettoeinkommensanteile der zehn Einkommensdezile in Deutschland im Jahr 2016 sowie Referenzwerte einer linearen Einkommensverteilung (D10: D1 =12:1) (Post-tax national income is the sum of primary incomes over all sectors (private and public), minus taxes. The base unit is the individual over age 20 (rather than the household), but resources are split equally within couples. Pre-tax national income is the sum of all pre-tax personal income flows accruing to the owners of the production factors, labor and capital, before taking into account the operation of the tax/transfer system, but after taking into account the operation of the pension system). (Quelle: World Inequality Database (WID.world): Post-tax und pre-tax national income, adults, equal split (shares), Abruf am 05.01.2020)

hinzu. Dazu muss ein Vielfaches zwischen dem ersten und zehnten Dezil festgelegt werden. Für diese Verhältniszahl lässt sich kaum ein rationales Kriterium festlegen. Auch ein subjektives Empfinden über eine faire Verhältniszahl dürfte innerhalb der Gesellschaft stark variieren. Die Platon'sche Verteilungsnorm eines maximal vierfachen Einkommens (bzw. Vermögens) würde in der heutigen Zeit sicher als zu eng angesehen werden; es lässt sich auch aus den oben bereits angesprochenen Gründen nicht in die heutige Zeit übertragen. Für die folgenden Überlegungen soll die der Schweizer Volksabstimmung zur Begrenzung der Managergehälter im Jahr 2013 zugrundeliegende Idee aufgenommen werden. Die Initiatoren dieser Volksabstimmung forderten, dass das Gehalt eines Topmanagers nicht mehr als das Zwölffache der Vergütung eines gering entlohnten Arbeiters (Mindestlohn) ausmachen solle. Dieses Verhältnis ergibt sich aus der Überlegung, dass der Bestverdienende einer Gesellschaft in einem Monat nicht mehr verdienen sollte, als ein Geringverdiener in einem Jahr erhält. ${ }^{17}$ Das Verhältnis zwischen dem untersten und dem obersten Dezil soll demzufolge dem Faktor zwölf entsprechen.

Abb. 5 zeigt eine solche Verteilung und stellt sie der 2016 in Deutschland existierenden tatsächlichen anteilsmäßigen Verteilung der Nettoeinkommen auf Dezile gegenüber. Bei der Idealverteilung wurde für das Nettoeinkommen des ersten Dezils beispielhaft ein Wert von 1000,- Geldeinheiten angenommen. Dementsprechend er-

17 In der historischen Satzung der Carl-Zeiss-Werke (Jena) war festgelegt, dass die Höhe des Einkommens der Führungskräfte auf das Zehnfache des mittleren Arbeitereinkommens zu beschränken sei (Carl-ZeissStiftung 1896). 
hält das zehnte Dezil 12.000,-- Geldeinheiten. Die Einkommensdifferenz zwischen den einzelnen Dezilen beträgt jeweils 1200,- Geldeinheiten. Das gesamte zur Verteilung zur Verfügung stehende Einkommen beträgt dann 65.000,- Geldeinheiten.

Um eine solche Dezilsverteilung zu erreichen, wären mit Sicherheit substanzielle Eingriffe in den Marktprozess notwendig, um bereits die Primärverteilung entsprechend zu beeinflussen (zum Beispiel Mindest- und Maximalverdienste). ${ }^{18}$ Alternativ oder zusätzlich wäre ein größeres Ausmaß an Einkommensumverteilung erforderlich. Dies ist angesichts der Höhe, die das zehnte Dezil gegenwärtig im Vergleich zum Normwert der Referenzverteilung hat, nur durch deutlich höhere Spitzensteuersätze zu erreichen. Da dies vor allem durch die von Platon formulierte Maxime motiviert wäre, den Abstand zwischen den höchsten und niedrigsten Einkommen zu begrenzen, ließe sich eine solchermaßen motivierte Reichensteuer als Platon-Steuer bezeichnen.

\section{Ansatzpunkte der Verteilungspolitik}

In diesem Abschnitt sollen schließlich einige konkrete Vorschläge zur Verteilungspolitik präsentiert und diskutiert werden, die auf unterschiedlichen Stufen im Produktionsprozess ansetzen. Neben verteilungspolitischen Interventionen in die Primär-

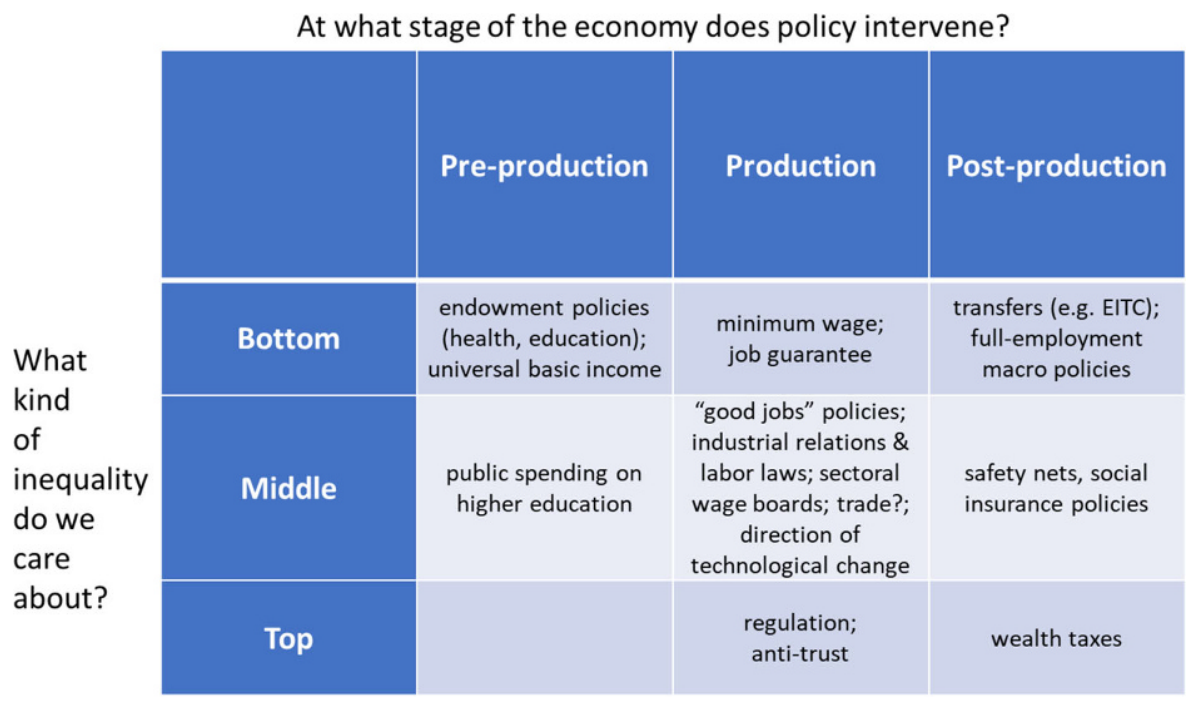

Abb. 6 3×3-Matrix verteilungspolitischer Interventionsmöglichkeiten

18 Der frühere Vorstandsvorsitzende der SAP, Bill McDermott, verdiente im Jahr 2019 15.176.900€. Nach Berechnungen der Süddeutschen Zeitung vom 27.02.2020 entspricht das etwa 3466,- $€$ pro Stunde. Dabei wurden 365 Arbeitstage und ein durchschnittlicher 12-Stunden-Arbeitstag unterstellt. Berücksichtigt man, dass Bill McDermott bereits Anfang Oktober von seinem Posten zurücktrat, errechnet sich ein Stundenlohn von $4456 €$. Dies entspricht dem 476-fachen des gesetzlichen Mindestlohns. 
und Sekundärverteilung werden zusätzlich noch verteilungspolitische Maßnahmen zur Verbesserung der Ausstattungen der Akteure in der Ökonomie behandelt. Dabei folge ich einem Schema, das kürzlich von Blanchard und Rodrik (2019, 2021) aufgestellt wurde. Die verschiedenen Ebenen, an denen verteilungspolitische Interventionen ansetzen können, werden in die Bereiche Pre-Production, Production und Post-Production eingeteilt. Außerdem unterscheiden die Autoren verteilungspolitische Interventionen danach, ob sie darauf abzielen, die Ungleichheit an der Spitze der Verteilung (top), am Ende der Verteilung (bottom) oder in der Mitte der Verteilung (middle) zu beeinflussen. Abb. 6 stellt eine $3 \times 3$-Matrix verteilungspolitischer Interventionsmöglichkeiten dar, die von Blanchard und Rodrik (2019) entwickelt wurde.

Der Bereich Pre-Production zielt vor allem auf die Ausstattung der Akteure ab. Blanchard und Rodrik geht es hier sowohl um eine gute Bildungspolitik als auch um eine bessere Gesundheitspolitik, die vor allem die unteren Schichten der Bevölkerung befähigt, in ihrem Berufsleben höhere Einkommen zu erzielen. Für die Mittelschicht in den USA ist es ein großes Problem, die in den letzten Jahrzehnten real enorm gestiegenen Ausbildungskosten ihrer Kinder zu finanzieren. Vor allem zur Finanzierung der Studiengebühren sind heute bereits Mittelstandsfamilien auf Kredite angewiesen, die diese Familien auf Jahre hinaus stark belasten. Blanchard und Rodrik sprechen sich daher für einen größeren Finanzierungsanteil der öffentlichen Hand in der Bildung aus.

In der Produktionssphäre (production stage) wird eine deutliche Anhebung des allgemeinen Mindestlohns befürwortet, nicht zuletzt, weil sich dieser in den USA in den letzten Jahren real substanziell verringert hat. Außerdem können sich die Autoren Arbeitsplatzgarantien seitens des Staates vorstellen. Von beiden Maßnahmen würden vor allem Angehörige der unteren Schichten profitieren. Die Mittelschicht könnte ihre Einkommensposition stärken, wenn ausreichend gut bezahlte Arbeitsplätze zur Verfügung stünden. Diese erhoffen sich Blanchard und Rodrik durch das, was sie „good jobs policies“ nennen. Hierfür sollen bessere Möglichkeiten der Arbeitnehmervertretungen beitragen. Ausdrücklich wird hier die deutsche Mitbestimmung als vorbildhaft genannt. Ein wichtiger Punkt für Blanchard und Rodrik ist auch die Steuerung des technischen Fortschritts durch den Staat. Sie sprechen sich dafür aus, beschäftigungsfreundliche Technologien zu fördern und Technologien, die Arbeitsplätze abbauen, mit Steuern zu belegen, um für die Privatwirtschaft entsprechende Anreizeffekte hinsichtlich der Richtung des technischen Fortschritts zu setzen. Der technische Fortschritt in der Digitalwirtschaft hat zu Winner-take-allMärkten geführt, wodurch Monopole und Quasi-Monopole entstanden sind. Hier habe der Staat eine wichtige Regulierungsaufgabe. Die Wettbewerbspolitik tue sich aber immer noch schwer, eine geeignete Antwort auf die neu entstandenen Monopole in der Digitalwirtschaft zu finden. ${ }^{19}$

\footnotetext{
19 In diese Kategorie gehören auch die Vorschläge von Freeman (2020, S. 2), dem es unter anderem um die Stärkung der gewerkschaftlichen Verhandlungsposition durch Interventionen der Politik geht. Er hebt die Bedeutung von Gewerkschaften bei der Vermeidung von Ungleichheit hervor: ,[...] unions have historically been the only institutional force in capitalist economies able to limit inequality and assure workers share in a growing economy. The inverse relation between union density and inequality is one of the stron-
} 
Im Bereich der Post-Production sind Maßnahmen der Umverteilung von Einkommen und Vermögen angesiedelt. Für die unteren Einkommensschichten sind zur Vermeidung von Armut und zur Verringerung der Einkommensungleichheit Transfereinkommen von großer Bedeutung. In den USA spielt wie in einigen anderen angelsächsischen Ländern der Earned Income Tax Credit (EITC), eine Art Steuergutschrift auf Arbeitseinkommen, eine bedeutsame Rolle. Dieser sollte nach Blanchard und Rodrik (2019) ausgebaut werden. Außerdem weisen sie darauf hin, dass auch makroökonomische Politikmaßnahmen, die auf eine Erhöhung von Wachstum und Beschäftigung abzielen, einen wichtigen Beitrag zur Reduktion von ökonomischer Ungleichheit leisten können, da zum Beispiel Vollbeschäftigung eine wichtige Voraussetzung für eine größere Gleichverteilung von Einkommen darstellt. Für die Mittelschicht seien gut entwickelte soziale Sicherungssysteme notwendig, um Ungleichheiten zu verringern. Auch hier wird auf die europäischen Wohlfahrtsstaaten verwiesen, die für die US-Politik ein Vorbild sein sollten. Schließlich halten Blanchard und Rodrik Vermögenssteuern aus verschiedenen Gründen für ein geeignetes Instrument, um Ungleichheiten zu verringern. Erstens könnten hieraus Einnahmen generiert werden, die für Ungleichheit reduzierende Ausgaben an anderer Stelle genutzt werden könnten. Zweitens könnten Vermögenssteuern einen Beitrag dazu leisten, politische Macht, die häufig mit hohem Reichtum einhergeht, zu eliminieren oder wenigstens zu begrenzen. Und drittens könnten Vermögenssteuern gerechtfertigt sein, wenn die Existenz von Reichtum das Ergebnis von Ungleichheiten sei, die an anderen Stellen bestehen würden.

In einer Abwägung aller hier vorgestellten verteilungspolitischen Interventionsmöglichkeiten kommen Blanchard und Rodrik (2019) und Rodrik (2019a, 2019b) zum Ergebnis, dass sie sich vor allem von Eingriffen in die Produktionssphäre (production stage) die größtmöglichen Erfolge für eine Reduktion der Ungleichverteilung versprechen. Ihre Ansichten befinden sich damit in einem deutlichen Kontrast zur bisher vertretenen, konventionellen Sichtweise, wonach verteilungspolitisch motivierte Eingriffe in Marktprozesse weitgehend vermieden werden sollten.

Die einzelnen Felder der $3 \times 3$-Matrix wurden im Hinblick auf die Situation und die Institutionen in den USA ausgefüllt. ${ }^{20}$ Die Vorschläge des bedeutenden britischen Ungleichheitsforschers Anthony Atkinson zur Änderung der Primärverteilung, die er in seinem letzten Buch (2015, Kap. 4-6) vorgestellt hat, werden von Hartwig und Krämer (2018, S. 53-55) zusammengefasst und im Folgenden leicht gekürzt wiedergegeben. Die Vorschläge von Atkinson beziehen sich im Wesentlichen auf die Situation in Großbritannien, wo die Ungleichheit der Einkommensverteilung größer ist als in Deutschland. ${ }^{21}$ Atkinson schlug konkret drei Bereiche vor, die die Beeinflussung der Primärverteilung betreffen.

Erstens sprach sich Atkinson für eine staatliche Beschäftigungs- und Lohnpolitik aus. Er trat in seinem Buch dafür ein, dass der Staat ein explizites Beschäftigungs-

gest empirical regularities in economics [...]“ Vgl. die weiteren Beiträge hierzu in Peterson Institute for International Economics (2019) beziehungsweise in Blanchard und Rodrick (2021).

$20 \mathrm{Zu}$ Vorschlägen, die sich auf Deutschland beziehen, vgl. Horn (2018, S. 96-97) und die darin aufgeführte Literatur.

21 Atkinson machte seine konkreten Vorschläge mit dem Ziel, den britischen Gini-Koeffizienten der Ungleichheit der Nettoeinkommen auf das Niveau von Deutschland abzusenken. 
ziel formuliert und, um dieses zu erreichen, notfalls auch als employer of last resort auftritt. Die Verhandlungsmacht der Gewerkschaften solle durch ein gesetzliches empowerment der Arbeitnehmerorganisationen gestärkt werden. Atkinson stellte sich dafür eine Art „konzertierte Aktion“ vor, die die Schaffung von Vertretungen gleich starker Sozialpartner beinhaltet. Diese würden auch die Aufgabe übertragen bekommen, Mindest- und Höchstlöhne zu bestimmen. Des Weiteren sah Atkinson auch die Wettbewerbspolitik in der Pflicht, die Verteilung der Einkommen zu beeinflussen. So solle die Wettbewerbspolitik auch die Auswirkungen von Unternehmenszusammenschlüssen auf die primäre Einkommensverteilung mitberücksichtigen. In den USA hat die erhöhte Unternehmenskonzentration in einigen Bereichen die markups und damit auch die jeweilige Gewinnquote gesteigert.

Zweitens forderte Atkinson eine breitere Streuung des Kapitalbesitzes in der Bevölkerung. Die Regierung solle Staatsanleihen mit einer garantierten positiven Realverzinsung für Kleinsparer emittieren. Außerdem forderte Atkinson, einen Staatsfonds aufzubauen, der in die Wirtschaft investiert und dessen Erträge an alle Bürger ausgeschüttet werden sollten. Besonders weitreichende Folgen dürfte sein diesbezüglicher Vorschlag haben, jedem Menschen, der im Land aufgewachsen ist, bei Erreichen des Erwachsenenalters ein Mindesterbe auszuzahlen. Dieses würde aus allen Erbschaften des laufenden Jahres stammen, die dementsprechend oberhalb eines bestimmen Freibetrags vollständig an einen gesellschaftlichen Erbschaftsfonds abzuführen wären. Diese radikale Maßnahme stellt aus Atkinsons Sicht die einzige effektive Möglichkeit dar, um der zunehmenden Vermögenskonzentration Einhalt zu gebieten. ${ }^{22}$

Das dritte Bündel von Maßnahmen betrifft die Steuerung des technischen Fortschritts - ein Handlungsfeld, das auch Blanchard und Rodrik (2019) für besonders wichtig erachten. Atkinson wies darauf hin, dass der Staat mit seiner Forschungsförderung den Innovationsprozess beeinflusst. Die Entwicklung von autonomen Fahrzeugen basiert nicht unwesentlich auf militärischer Forschung, die das Ziel verfolgte, Soldaten in lebensgefährlichen Einsätzen zu schützen. In der Folge könnte die zivile Anwendung, etwa bei führerlosen Lastkraftwagen, dazu führen, dass eine große Zahl von Arbeitsplätzen für LKW-Fahrer entfällt. Nach Atkinson haben Politiker eine Verantwortung für die potentiellen Auswirkungen von Innovationen auf die Beschäftigung. Innovationen, die in großem Umfang Arbeitslosigkeit verursachen können, sollten nicht mehr durch Subventionen und andere Unterstützungsmaßnahmen gefördert werden, da Arbeitslosigkeit unmittelbar zu einer größeren Ungleichverteilung der Einkommen führt. Problematisch hieran dürfte allerdings sein, dass die Beschäftigungsauswirkungen von Innovationen ex ante nur schwer einzuschätzen sind und damit das ohnehin schwache Produktivitätswachstum weiter reduziert werden könnte.

\footnotetext{
22 Die Besteuerung von Erbschaften steht auch in der Tradition der liberalen Staatsphilosophie. Oben wurde bereits darauf hingewiesen, dass sich John Stuart Mill für substantielle Vermögens- und Erbschaftssteuern ausgesprochen hat, um Machtkonzentrationen in Wirtschaft und Gesellschaft zu verhindern. Auch Sohmen (1976), der ansonsten die negativen allokativen Effekte von Umverteilung hervorhob, bezeichnete eine ,weitgehende Beschränkung des Erbrechts“ als ,zweifellos die natürlichste Methode zur Verwirklichung“ des Ziels ,einer gleichmäßigeren Verteilung der politischen Macht und als Folge davon (...) einer ausgeglichenen Verteilung aller übrigen Rechte und Pflichten in der Gesellschaft“ (ebd., S. 374-5).
} 
Was den technischen Fortschritt betrifft, könnte daher mit Blick auf den hybriden öffentlich-privaten Charakter der hier betrachteten Wirtschaftsaktivitäten eine Umverteilungspolitik besser geeignet sein. Schließlich hat das Geld der Steuerzahler häufig dazu beigetragen, die Entwicklung neuer Technologien zu finanzieren und den Weg für neue und sehr erfolgreiche Unternehmen zu ebnen - die sog. GAFAUnternehmen sind ein Beispiel dafür. Es wäre nur gerecht, wenn der Steuerzahler auch an den Gewinnen, die sie erzielen, angemessen beteiligt würde. Andernfalls könnte der Steuerzahler zu Recht einwenden, dass die Kosten von Innovationen systematisch sozialisiert, die Gewinne aber privatisiert würden.

Atkinson macht in den danach folgenden Kapiteln noch eine Reihe von Vorschlägen zur klassischen Umverteilungspolitik. Er tritt dafür ein, die Bemessungsgrundlage der Einkommenssteuern durch eine Begrenzung von Abzugsmöglichkeiten zu verbreitern und den Spitzensteuersatz auf $65 \%$ zu erhöhen. Dagegen sollte es Steuerrabatte für geringfügige Arbeitseinkommen geben. Wesentlich progressiver als bisher sollten Steuern auf Erbschaften und Grundbesitz ausgestaltet werden. Bei den Transfereinkommen setzt sich Atkinson für ein deutlich erhöhtes Kindergeld und für ein Bürgergeld ein, das alle diejenigen erhalten sollten, die einen Beitrag für die Gesellschaft leisten.

\section{Schlussbemerkungen}

Im Vergleich zu den angelsächsischen Ländern, in denen die wirtschaftliche und soziale Ungleichheit zum Teil bereits extreme Ausmaße erreicht hat, ist die Einkommensungleichheit in Deutschland deutlich geringer ausgeprägt, aber auch hier hat sie sich in den letzten 25 Jahren spürbar erhöht (OECD 2015). Der Trend zu einer steigenden Einkommensungleichheit hat sich in der jüngeren Vergangenheit zwar deutlich abgeschwächt, allerdings ist trotz der seit geraumer Zeit vergleichsweise guten Lage auf dem Arbeitsmarkt keineswegs eine Trendumkehr in Richtung größerer Gleichheit erfolgt.

Es gibt ausreichend Anlass davon auszugehen, dass die Kräfte, die in den letzten Jahrzehnten zu einer weiteren Polarisierung der Einkommensverteilung geführt haben, auch in Zukunft weiterwirken werden. Milanović (2016, S. 180 f.) diskutiert eine Reihe von Faktoren, die dafür verantwortlich sind, darunter eine aus verschiedenen Gründen weiterhin wachsende Gewinnquote sowie die hohe Konzentration der Kapitaleinkommen bei den oberen Einkommensgruppen. Hinzu kommt, dass die wachsende Konzentration der Einkommen den besser gestellten Schichten einen erhöhten Einfluss auf die Politik verleiht. Aus gutem Grund zitierte bereits Adam Smith zustimmend den Staatstheoretiker und Philosophen Thomas Hobbes: „Wealth, as Mr. Hobbes says, is power" (Smith 2012 [1776], I.v. 3). Damit meinte Smith nicht nur politische Macht, aber diese eben auch. Dem wachsenden Zusammenhang von Reichtum und politischer Macht können sich auch die westlichen Demokratien immer weniger entziehen. ${ }^{23}$

\footnotetext{
23 Milanović (2016, S. 192-211) sieht vor allem für die USA die Gefahr, dass sich das politische Sys-
} tem hin zu einer Plutokratie entwickelt. Für Europa erwartet er aufgrund der andersartigen Institutionen 
Es kann daher vermutet werden, dass Maßnahmen, die eine Umverteilung von Einkommen und Vermögen zugunsten der ärmeren Schichten bewirken, immer schwerer durchzusetzen sein werden, je mehr der Einkommensanteil der reichen Haushalte und damit ihr politischer Einfluss wächst. Dies betrifft nicht nur die monetäre Umverteilung, sondern auch die Umverteilung, die durch die Bereitstellung von öffentlichen Dienstleistungen (d.h. Realtransfers) erfolgt. ${ }^{24}$

Verteilungspolitische Maßnahmen werden in der Regel insbesondere mit der Begründung abgelehnt, dass sie den Markt und dessen Effizienz beeinträchtigen. Dies betrifft insbesondere Eingriffe in die Primärverteilung. Der ideale Wettbewerbsmarkt ist in der Lage, eine unter Wohlfahrtsgesichtspunkten pareto-optimale Allokation der Ressourcen zu erreichen. Jegliche staatliche Eingriffe in Marktprozesse werden daher als wohlfahrtreduzierend angesehen, da diese die Allokation der Ressourcen „stören“. Hervorgehoben wird, dass von staatlichen Interventionen mit dem Ziel, die Primär- oder auch die Sekundärverteilung zu verändern, negative Anreizeffekte ausgehen, die den „Kuchen“, der zur Verteilung zur Verfügung steht, kleiner machten. In dieser Perspektive besteht ein unausweichlicher Trade-off zwischen Gleichheit und Effizienz (Okun 1975; Furman 2016). Gisela Kubon-Gilke (2013, S. 160) weist jedoch zurecht darauf hin: ,Sollten Märkte nicht perfekt funktionieren oder sollte es möglich sein, eine Umverteilung unabhängig vom Marktmechanismus zu finden, dann gilt dieses Argument nicht mehr allgemein, und es ist notwendig, im Einzelfall zu überprüfen, wie die einzelnen Maßnahmen die Allokation und die Verteilung beeinflussen“. Angesichts dessen können die Effizienzeigenschaften, die üblicherweise perfekten Märkten zugeschrieben werden, nicht als Argument gegen eine in der Realität stattfindende Politik der Einkommens- und Vermögensverteilung angeführt werden.

Wenn man das Ziel verfolgt, dass die Einkommen und Vermögen gleichmäßiger verteilt sein sollen als dies gegenwärtig der Fall ist, erscheint ein multi-instrumenteller Ansatz in der Verteilungspolitik im Sinne von Blanchard und Rodrik (2019) notwendig zu sein, da die klassische Umverteilungspolitik hierfür wahrscheinlich nicht ausreicht und außerdem teilweise an ihre Grenzen stößt. Die Warnungen bezüglich potenziell schädlicher Allokationseffekte von Umverteilungen bzw. von staatlichen Eingriffen in den Marktprozess haben zwar ohne Frage ihre grundsätzliche Berechtigung (Stichwort: Anreizeffekte). Sie sind aber in der Vergangenheit zu oft als Argument dafür benutzt worden, um verteilungspolitische Maßnahmen zu beschränken oder ganz zu unterbinden. Angesichts der zu Recht hinterfragbaren theoretischen Basis, mit der verteilungspolitische Maßnahmen häufig abgelehnt werden, neuerer empirische Evidenz und des zunehmenden Handlungsdrucks in der Verteilungsfrage sollten derartige Einwände auch Ökonominnen und Ökonomen nicht davon abhalten, grundsätzlich über eine Vielzahl von verteilungspolitischen Maßnahmen nachzudenken, um die Einkommen und Vermögen wieder gleicher zu verteilen. Auf diesem Gebiet hat sich die Ökonomik in den letzten Jahrzehnten zu häufig als eine Wissenschaft erwiesen, die der Politik aufzeigt, was alles bei verteilungspolitischen

des politischen Systems und der Bedeutung der Immigration eher eine Hinwendung zu Populismus und (Wirtschafts-)Nationalismus. In den USA sind aktuell sogar beide Tendenzen zu beobachten.

24 Zum politischen Prozess der Umverteilung vgl. Windhoff-Héritier (1982, S. 78 ff.). 
Interventionen schädlich und unnütz ist, und dadurch zu ihrem Ruf, eine ,dismal science“ zu sein, beigetragen. Sie sollte sich wieder stärker darum bemühen, der Politik Handreichungen zu geben und geeignete Wege aufzuzeigen, mit denen es möglich wird, eine drängende gesellschaftspolitische und ökonomische Aufgabe wirksam anzugehen. Die grundsätzliche Stärke der Wirtschaftswissenschaften ist es, dabei Kosten und Nutzen von alternativen Maßnahmen zu Zielerreichung aufzuzeigen. Daher ist die Behandlung von Fragen von Wachstum und Verteilung unter Einbeziehung von normativen Überlegungen dringender denn je.

Danksagung Ich danke Peter Kalmbach für seine hilfreichen Kommentare zu einer früheren Fassung meines Textes sowie den Teilnehmerinnen und Teilnehmern der Jahrestagung 2020 des wirtschaftspolitischen Ausschusses des Vereins für Socialpolitik in Berlin für ihre Anmerkungen zu meinem Referat.

Funding Open Access funding enabled and organized by Projekt DEAL.

Open Access Dieser Artikel wird unter der Creative Commons Namensnennung 4.0 International Lizenz veröffentlicht, welche die Nutzung, Vervielfältigung, Bearbeitung, Verbreitung und Wiedergabe in jeglichem Medium und Format erlaubt, sofern Sie den/die ursprünglichen Autor(en) und die Quelle ordnungsgemäß nennen, einen Link zur Creative Commons Lizenz beifügen und angeben, ob Änderungen vorgenommen wurden.

Die in diesem Artikel enthaltenen Bilder und sonstiges Drittmaterial unterliegen ebenfalls der genannten Creative Commons Lizenz, sofern sich aus der Abbildungslegende nichts anderes ergibt. Sofern das betreffende Material nicht unter der genannten Creative Commons Lizenz steht und die betreffende Handlung nicht nach gesetzlichen Vorschriften erlaubt ist, ist für die oben aufgeführten Weiterverwendungen des Materials die Einwilligung des jeweiligen Rechteinhabers einzuholen.

Weitere Details zur Lizenz entnehmen Sie bitte der Lizenzinformation auf http://creativecommons.org/ licenses/by/4.0/deed.de.

\section{Literatur}

Alvaredo, F., Atkinson, A.B., Piketty, T., Saez, E., \& Zucman, G. (2020). World wealth and income database. URL: WID. World.

Arndt, C., Dann, S., Kleimann, R., Strotmann, H., \& Volkert, J. (2006). Das Konzept der Verwirklichungschancen (A. Sen). Empirische Operationalisierung im Rahmen der Armuts- und Reichtumsmessung. Machbarkeitsstudie (Endbericht). Bonn: Bundesministerium für Arbeit und Soziales.

Atkinson, A.B. (2015). Inequality. What can be done? Cambridge (MA), London: Harvard University Press.

Badelt, C., \& Österle, A. (2001). Grundzüge der Sozialpolitik. Allgemeiner Teil: Sozialökonomische Grundlagen (2. Aufl.). Wien: Manz.

Basu, K. (2011). Beyond the invisible hand. Groundwork for a new economics. Princeton, Oxford: Princeton University Press.

Blanchard, O., \& Rodrik, D. (2019). We have the tools to reverse the rise in inequality. Reflections on the conference on combating inequality: rethinking policies to reduce inequality in advanced economies, Peterson institute for international economics, october 17-18, 2019. https://www.piie.com/ commentary/speeches-papers/we-have-tools-reverse-rise-inequality. Zugegriffen: 1. Dez. 2019.

Blanchard, O., \& Rodrik, D. (Hrsg.). (2021). Combating inequality: rethinking government's role. Cambridge (MA) und London: MIT Press (im Erscheinen).

von Böhm-Bawerk, E. (1914). Macht oder ökonomisches Gesetz? Zeitschrift für Volkswirtschaft, Sozialpolitik und Verwaltung, 23, 205-271.

Carl-Zeiss-Stiftung (1896). Statut der Carl-Zeiss-Stiftung. Jena: Carl Zeiss.

Carlsson, F., Daruvala, D., \& Johansson-Stenman, O. (2005). Are people inequality-averse, or just riskaverse? Economica, 72(287), 375-396. 
Clark, J.B. (1927) [1899]. The distribution of wealth: a theory of wages, interest and profits. New York: Macmillan.

Deutscher Bundestag (2005). Unterrichtung der Bundesregierung: Lebenslagen in Deutschland. Zweiter Armuts- und Reichtumsbericht. Drucksache 15/5015, 3.3.2005. Berlin: Deutscher Bundestag.

Easterlin, R. A. (1973). Does money buy happiness? The Public Interest, 30, 3-10.

Easterlin, R. A. (1995). Will raising the incomes of all increase the happiness of all? Journal of Economic Behavior \& Organization, 27(1), 35-47.

Edgeworth, F. Y. (1904). The theory of distribution. The Quarterly Journal of Economics, 18(2), 149-219.

Fehr, E., \& Schmidt, K. M. (1999). A theory of fairness, competition, and cooperation. Quarterly Journal of Economics, 114(3), 817-886.

Felbermayr, G., Battisti, M., \& Lehwald, S. (2016). Einkommensungleichheit in Deutschland, Teil 2: Die Rolle der Umverteilung. ifo Schnelldienst, 69(13), 28-37.

Fratzscher, M. (2016). Verteilungskampf. München: Hanser.

Freeman R.B. (2020): Ownership Cures for Inequality. https://scholar.harvard.edu/files/freeman/files/ ownership_cures_for_inequality_freeman_ms-for-dash_24mar2020.docx. Zugegriffen: 1. Juni 2020. Erscheint in: O. Blanchard \& D. Rodrik (2021) (Hrsg.), Combating Inequality: Rethinking Policies to Reduce Inequality in Advanced Economies, Cambridge (MA): MIT Press (im Erscheinen).

Furman, J. (2016). Equality and efficiency: a global perspective. World Bank group macro-economics and fiscal management global practice annual forum May 2, 2016. Washington, D.C.: The World Bank. https://obamawhitehouse.archives.gov/sites/default/files/page/files/20160502_wb_mfm_forum_cea. pdf. Zugegriffen: 6. Jan. 2020.

Gaertner, W. (2009). Amartya Sen (*1933). In H.D. Kurz (Hrsg.), Klassiker des ökonomischen Denkens (Bd. 2, S. 354-372). München: C.H. Beck.

Gawel, E. (2009). Grundzüge der mikroökonomischen Theorie. Lohmar, Köln: Eul-Verlag.

Hartwig, J. (2018). Wachstumsfolgen von Einkommensungleichheit. Theorie, empirische Evidenz und Politikempfehlungen. List Forum, 44, 101-141.

Hartwig, J., \& Krämer, H. (2018). Wer kann sich Dienstleistungen zukünftig noch leisten? Über die Folgen des Zusammenhangs von Einkommensverteilung und Strukturwandel. In H. Gischer, J. Hartwig \& B. Sahin (Hrsg.), Bewegungsgesetze des Kapitalismus (S. 35-59). Marburg: Metropolis.

Hayek, F.A. (1996) [1981]. Ungleichheit ist nötig, wiederabgedruckt in Wirtschaftswoche, 11.1.1996, 16.

Hayek, F.A. (2006) [1960]. The constitution of liberty. London, New York: Routledge.

Helmedag, F. (2010). Fair wages and social welfare. Journal of Income Distribution, 19(3-4), 3-8.

Horn, G. A. (2018). Wie entstand die Ungleichheit der Einkommen in Deutschland und was sollte man dagegen tun? List Forum, 44, 77-99.

ILO, IMF, OECD, \& World Bank Group (2015). Income inequality and labour income share in G20 countries: trends, impacts and causes. Paper prepared for the G20 Labour and Employment Ministers and Joint Meeting with the G20 Finance Ministers, Ankara, 3-4. 2015.

Klodt, H. (2018). Wirtschaftspolitik und Verteilung: Editorial. List Forum, 44, 73-76.

Krämer, H. (2014). Ungleichheit, Marktversagen und Verteilungsnormen. In M. Held, G. Kubon-Gilke \& R. Sturn (Hrsg.), Unsere Institutionen in Zeiten der Krisen. Jahrbuch Normative und institutionelle Grundfragen der Ökonomik, (Bd. 13, S. 99-126). Marburg: Metropolis.

Krämer, H. (2017). Reichtum in den ökonomischen Theorien. In N. Dimmel, J. Hofmann, M. Schenk \& M. Schürz (Hrsg.), Handbuch Reichtum. Neue Erkenntnisse aus der Ungleichheitsforschung (S. 118-137). Innsbruck, Wien, Bozen: StudienVerlag.

Krämer, H. (2019). Das klassische Konzept der unproduktiven Arbeit und seine Folgen für das moderne Verständnis von Dienstleistungen. In H.-M. Trautwein (Hrsg.), Neue Perspektiven auf die Politische Ökonomie von Karl Marx und Friedrich Engels. Die Marx-Engels-Gesamtausgabe. Schriften des Vereins für Socialpolitik, Neue Folge, Bd. 115, Studien zur Entwicklung der ökonomischen Theorie, Bd. 34. (S. 113-175). Berlin: Duncker \& Humblot.

Kubon-Gilke, G. (2013). Außer Konkurrenz. Sozialpolitik im Spannungsfeld von Markt, Zentralsteuerung und Traditionssystemen (2. Aufl.). Marburg: Metropolis.

Kumhof, M., \& Ranciere, R. (2010). Inequality, leverage and crises. IMF Working Papers 268. Washington, D.C.: International Monetary Fund.

Kurz, H.D. (2002). Surplus, Abstinenz und Lohnfonds. John Stuart Mill zur Theorie der Einkommensverteilung. In E. W. Streissler (Hrsg.), John Stuart Mill. Studien zur Entwicklung der ökonomischen Theorie XIX. (S. 253-280). Berlin: Duncker \& Humblot.

Kurz, H. D. (2013). Geschichte des ökonomischen Denkens. München: Beck.

Kurz, H.D. (2018). Hin zu Marx und über ihn hinaus, Perspektiven der Wirtschaftspolitik, 19(3), 245-265. 
Mankiw, N.G. (2010). Spreading the wealth around: reflections inspired by Joe the Plumber. Eastern Economic Journal, 36(3), 285-298.

Mankiw, N.G. (2013). Defending the one percent. Journal of Economic Perspectives, 27(3), 21-34.

Marshall, A. (1920). Principles of economics (8. Aufl.). London: Macmillan.

Marx, K. (1962-1964) [1867-1894]. Das Kapital. 3 Bände, MEW Bd. 23-25, Berlin: Dietz.

Milanović, B. (2016). Global inequality. A new approach for the age of globalization. Cambridge (MA): Bellknap Press of Harvard University Press.

Mill, J.St. (2016) [1848]. Grundsätze der Politischen Ökonomie. Teilband 1. Marburg: Metropolis. Principles of Political Economy with some of their Applications to Social Philosophy, 7. Aufl. Zitiert nach: Library of Economics and Liberty. https://www.econlib.org/library/Mill/mlP.html. Zugegriffen: 20. Febr. 2020.

Musgrave, R. A. (1957). A multiple theory of budget determination. Finanzarchiv, 17, 333-343.

Musgrave, R. A. (1959). The theory of public finance. New York: McGraw-Hill.

Musgrave, R. A., Musgrave, P. B., \& Kullmer, L. (1994). Die öffentlichen Finanzen in Theorie und Praxis (6. Aufl.). Bd. 1. Tübingen: UTB. nach: Musgrave, R.A. \& Musgrave P.B. (1989). Public Finance in Theory and Practice, 5. Aufl., New York: McGraw-Hill

Nozick, R. (1974). Anarchy, state, and utopia. New York: Basic Books.

OECD (2015). In it together. Why less inequality benefits all. Paris: OECD Publishing.

OECD (2020). Income distribution database. Version Juni 2019. http://stats.oecd.org/Index.aspx?DataSet Code=IDD. Zugegriffen: 3. Jan. 2020.

Okun, A.M. (1975). Equality and efficiency. The big trade-off. Washington, D.C.: Brookings Institution Press.

Ostry, J.D., Berg, A., \& Tsangarides, C. G. (2014). Redistribution, inequality, and growth. IMF staff discussion note 14/02. Washington, D.C.: International Monetary Fund.

Persson, T., \& Tabellini, G. (1994). Is inequality harmful for growth? American Economic Review, 84(3), $600-621$.

Peterson Institute for International Economics (2019). Conference combating inequality. Rethinking policies to reduce inequality in advanced economies. 17-18.10.2019. Washington, D.C.: Peterson Institute for International Economics.

Pigou, A.C. (1912). Wealth and welfare. London: Unwin.

Pigou, A.C. (1952) [1920]. The economics of welfare. 4. Aufl. London: Macmillan.

Piketty, T. (2014). Capital in the 21st century. Cambridge (MA): Bellknap Press of Harvard University Press.

Platon (1862). Nomoi (De legibus, Die Gesetze). Nach der Übersetzung von Dr. F. Susemihl, in: Platon's Werke, vierte Gruppe, neuntes bis fünfzehntes Bändchen. Stuttgart. http://www.opera-platonis.de/ Nomoi.html. Zugegriffen: 3. Jan. 2020.

Rawls, J. (1971). Theory of justice. Cambridge (MA): Bellknap Press of Harvard University Press.

Ricardo, D. (1951-1973) [1817]. On the principles of political economy and taxation. Hrsg. von P. Sraffa \& M.H. Dobb, The works and correspondence of David Ricardo. (11 Bände) Cambridge: Cambridge University Press.

Rodrik, D. (2019a). Closing presentation at the conference Combating Inequality. Rethinking Policies to Reduce Inequality in Advanced Economies. Peterson Institute for International Economics. 17-18.10.2019. Washington D.C.: Peterson Institute for International Economics.

Rodrik, D. (2019b). Tackling Inequality from the Middle. https://www.project-syndicate.org/commentary/ tackling-inequality-from-the-middle-by-dani-rodrik-2019-12. Zugegriffen: 12. Dez. 2019.

Sen, A. (1985). Commodities and capabilities. New Delhi: Oxford University Press.

Sen, A. (1999). Development as freedom. New York: Alfred Knopf.

Sen, A. (2000). Ökonomie für den Menschen. Wege zur Gerechtigkeit und Solidarität in der Marktwirtschaft. München, Wien: Carl Hanser Verlag.

Sen, A. (2009) [1973]. Ökonomische Ungleichheit. Marburg: Metropolis.

Sen, A. (2010) [2009]. Die Idee der Gerechtigkeit. München: Beck.

Smith, A. (2012) [1776]. Untersuchung über Wesen und Ursachen des Reichtums der Völker. 2 Bände, hrsg. und eingeleitet von E.W. Streissler, übersetzt von M. Streissler, Tübingen: Mohr Siebeck. Original: A. Smith (1976) [1776]. An Inquiry into the Nature and Causes of the Wealth of Nations. Glasgow edition, hrsg. von R.H. Campbell, A. S. Skinner \& W. B. Todd. Oxford: Oxford University Press.

Sohmen, E. (1976). Allokationstheorie und Wirtschaftspolitik. Tübingen: J.C.B. Mohr (Paul Siebeck).

Solow, R. M. (1956). A contribution to the theory of economic growth. Quarterly Journal of Economics, 70(1), 65-94. 
Spahn, H. P. (2002). Profit und Zins bei John Stuart Mill. In E. W. Streissler (Hrsg.), John Stuart Mill. Studien zur Entwicklung der ökonomischen Theorie XIX (S. 215-252). Berlin: Duncker \& Humblot.

Streissler, E. W. (1981). Adam Smith - ,der Adam“ oder ,nur Wachstum“? Paradoxa einer wissenschaftlichen Revolution. In F. Neumarck (Hrsg.), Studien zur Entwicklung der ökonomischen Theorie I. Schriften des Vereins für Socialpolitik, NF 115/I (S. 9-52). Berlin: Duncker und Humblot. Wiederabgedruckt in und zitiert nach: Adam Smith (2012) [1776]. Untersuchung über Wesen und Ursachen des Reichtums der Völker, 2 Bände, hrsg. und eingeleitet von E. W. Streissler, übersetzt von M. Streissler (S. 32-70). Tübingen: Mohr Siebeck.

Südekum, J. (2018). Digitalisierung und die Zukunft der Arbeit. Was ist am Arbeitsmarkt passiert und wie soll die Wirtschaftspolitik reagieren? IZA Standpunkte 90. Bonn: IZA-Institute of Labor Economics.

SVR (Sachverständigenrat zur Begutachtung der gesamtwirtschaftlichen Entwicklung) (2017). Jahresgutachten 2017/18. Wiesbaden: SVR.

Voitchovsky, S. (2009). Inequality and economic growth. In B. Nolan, W. Salverda \& T. M. Smeeding (Hrsg.), The Oxford handbook of economic inequality (S. 549-574). Oxford: Oxford University Press.

Wilkinson, R.G., \& Pickett, K. (2009). The spirit level. Why more equal societies almost always do better. London: Allen Lane.

Windhoff-Héritier, A. (1982). Soziale Gleichheit durch Umverteilung. Zum Begriff der Umverteilungspolitik und dessen politikwissenschaftlicher Einbindung. Politische Vierteljahresschrift, 23(1), 68-86. 